\title{
WestVirginiaUniversity
}

THE RESEARCH REPOSITORY @ WVU

Graduate Theses, Dissertations, and Problem Reports

1999

\section{Encapsulation of particles using brittle coatings for subterranean applications}

Aashish L. Bhatia

West Virginia University

Follow this and additional works at: https://researchrepository.wvu.edu/etd

\section{Recommended Citation}

Bhatia, Aashish L., "Encapsulation of particles using brittle coatings for subterranean applications" (1999). Graduate Theses, Dissertations, and Problem Reports. 1520.

https://researchrepository.wvu.edu/etd/1520

This Thesis is protected by copyright and/or related rights. It has been brought to you by the The Research Repository @ WVU with permission from the rights-holder(s). You are free to use this Thesis in any way that is permitted by the copyright and related rights legislation that applies to your use. For other uses you must obtain permission from the rights-holder(s) directly, unless additional rights are indicated by a Creative Commons license in the record and/ or on the work itself. This Thesis has been accepted for inclusion in WVU Graduate Theses, Dissertations, and Problem Reports collection by an authorized administrator of The Research Repository @ WVU. For more information, please contact researchrepository@mail.wvu.edu. 


\title{
Encapsulation Of Particles Using Brittle Coatings For Subterranean Applications
}

\author{
Aashish L. Bhatia
}

Thesis submitted to the

College of Engineering and Mineral Resources

at West Virginia University

in partial fulfillment of the requirements

for the degree of

Master of Science

in

Chemical Engineering

\author{
Richard Turton, Ph.D., Chair \\ John Zondlo, Ph.D. \\ Alfred Stiller, Ph.D.
}

Department of Chemical Engineering

\author{
Morgantown, West Virginia
}

1999

Keywords: encapsulation, brittle coating, particle coating, viscosity-reducer, breaker, subterranean, fracturing, ammonium persulfate, acrylate, silica, polyurethanes, agglomerates, Wurster coater, iodometric titration 


\section{ABSTRACT \\ Encapsulation Of Particles Using Brittle Coatings For Subterranean Applications}

\section{Aashish L. Bhatia}

The production of a brittle and moisture-resistant coating for particles, which reduce or "break" the viscosity of fracturing fluids, was explored.

The viscosity-reducer was ammonium persulfate. Polyurethane and acrylate coatings were investigated. The coated particles were assessed for dissolution in water before and after being subjected to a compression test. Polyurethane-coated particles had a high leach prior to compression and were considered unsuitable. Acrylate coatings gave much better results. The addition of significant quantities of silica in the coat increased significantly its brittleness. The acrylate formed agglomerates during the coating process. These were easier to break than single particles. These agglomerates also gave a low leach rate and large release upon compression.

Agglomerates coated with acrylate and silica provide a novel solution to the opposing constraints of high water resistance and high susceptibility to brittle fracture. 


\section{DEDICATION}

This research work is dedicated to

my late grandparents, Saraswati \& Khubchand Gajria

my grandma, Gopi Bhatia

my parents, Kavita and Lachman Bhatia

and my sister, Karuna 


\section{ACKNOWLEDGEMENTS}

First and foremost, I would like to thank my advisor Dr. Richard Turton for his help and advice throughout the course of this research. No words can adequately express my pleasure in working for a researcher of his caliber, and more importantly for such a fine human being. His methodical ways of analyzing things helped me overcome numerous obstacles on the way, and I owe the success of this work to him. Moreover, his suggestions during the write-up of this thesis were a boon.

I would also like to thank my committee members, Dr Al Stiller and Dr. John Zondlo, for their constructive criticism throughout the course of this work. Their suggestions helped me in looking at the work from a different perspective, and hence made it more comprehensive.

I would like to thank the technician, Jim Hall, without whose help, it would not be possible for any graduate student to do research in the Chemical Engineering Dept. He is one of the most patient people I've met, and I've never heard him complaining in spite of the heavy workload.

I would like to thank Halliburton Energy Services for their financial support and Dr. Lewis Norman for lending his expertise from time to time.

I would like to thank all my colleagues in the lab, Ravi Joshi, Kandis Sudsakorn, Huma Hakim, Rob Taylor, and Ganesh Subramaniam for making working in the lab an enjoyable experience. Special thanks to Ravi for helping my get started on the research, and Huma for being a constant source of support and encouragement.

I am also grateful to the following people for their help at various stages of this work: Dr. Stansberry and Sushant Agarwal for their help with the microscopic analysis, 
Katie Ziemer for her help with the SEM analysis, and Ed Wen \& Swetha Anegunta for their help with the Instron tester.

I would like to thank all my friends in Morgantown, for making this stay a very memorable one. I would especially like to thank Vikram Tolani, Rohit Borker and Iftekhar Kalsekar, for being the "friends in need, friends indeed".

Finally, I would like to thank my parents for bringing me up to where I am today. Mom, and Dad, I can never repay all that you've done for me. This dedication is a small gift to you. I hope I have made you proud. Last, but not the least, my sister, Karuna, who has grown up to be a fine young lady, but will always by my kid sister to me. Even though we are seas apart, you three are always on my mind, and will always be the guiding force of my life. Thank you!

- Aashish 


\section{TABLE OF CONTENTS}

Dedication $\quad$ iii

Acknowledgements $\quad$ iv

Table of Contents $\quad$ vi

$\begin{array}{ll}\text { List of Figures } & \text { ix }\end{array}$

List of Tables $\quad$ X

1. INTRODUCTION 1

2. LITERATURE REVIEW 4

$\begin{array}{ll}2.1 \text { INTRODUCTION } & 4\end{array}$

2.2 THE FRACTURING PROCESS 4

2.3 FRACTURING FLUIDS

2.4 METHODS FOR RELEASING FRACTURING FLUID BREAKERS 8

2.5 THE ENCAPSULATED BREAKER 11

$\begin{array}{ll}2.6 \text { COATING FORMULATION } & 12\end{array}$

2.6.1 Aqueous vs. Organic Coating 12

2.6.2 Coating Compositions 13

$\begin{array}{ll}\text { 2.6.2.1 Polyurethane Coatings } & 13\end{array}$

$\begin{array}{ll}\text { 2.6.2.2 Acrylate Coatings } & 14\end{array}$

$\begin{array}{ll}2.7 \text { THE COATING PROCESS } & 14\end{array}$

$\begin{array}{ll}\text { 2.7.1 The Fluid-Bed Equipment } & 15\end{array}$

2.7.2 Bottom Spray (Wurster) 18

3. EXPERIMENTAL EQUIPMENT AND PROCEDURES 21

$\begin{array}{ll}\text { 3.1 EXPERIMENTAL METHODOLOGY } & 21\end{array}$ 
$\begin{array}{ll}\text { 3.2 COATING FORMULATION } & 21\end{array}$

3.2.1 Polyurethane Coatings 22

3.2.2 Acrylate Coatings $\quad 22$

3.3 COATING PROCESS 23

$\begin{array}{ll}\text { 3.3.1 Polyurethane Coatings } & 23\end{array}$

3.3.2 Acrylate Coatings 23

3.3.2.1 Coating Procedure $\quad 26$

$\begin{array}{ll}\text { 3.3.2.2 Operating Conditions } & 26\end{array}$

3.4 TESTS FOR DETERMINATION OF COATING STRENGTH 30

3.4.1 Leach Test 30

3.4.2 Compression Test 30

3.4.3 Determination of Suitable Weight of Test Mixture to be Used 32

3.4.4 Procedure for Iodometric Titration 33

4. RESULTS AND DISCUSSIONS 37

4.1 POLYURETHANE COATINGS 37

$\begin{array}{ll}\text { 4.2 ACRYLATE COATINGS } & 39\end{array}$

4.2.1 Effect of Coating Level

4.2.2 Effect of Nozzle Size on the Size Distribution of Coated Particles

4.2.3 Release Rates for Coated Particles/Agglomerates of Different Sizes $\quad 44$

4.2.4 Effect of Curing $\quad 46$

4.2.5 Effect of Silica in Coat $\quad 48$

4.2.6 Release Rates for Agglomerates Coated with Optimum Formulation $\quad 51$

4.2.7 Performance of Agglomerates Compared with Commercial Breakers $\quad 54$ 
4.2.8 Mechanism of Formation and Crushing of Agglomerates 56

4.2.9 Effect of Change in Concentration of Cross-Linking Agent 61

4.2.10 Effect of Draft Tube Height During the Coating 63

5. SUMMARY \& SIGNIFICANCE OF WORK 64

5.1 SUMMARY OF WORK

5.2 SIGNIFICANCE OF WORK

6. RECOMMENDATIONS FOR FUTURE WORK 67

6.1 POLYURETHANE COATINGS

6.2 ACRYLATE COATINGS $\quad 67$

$\begin{array}{ll}6.3 \text { OTHER POLYMER SYSTEMS } & 68\end{array}$

Nomenclature \& Abbreviations $\quad 69$

$\begin{array}{ll}\text { References } & 70\end{array}$

$\begin{array}{ll}\text { Appendix } & 72\end{array}$ 


\section{List of Figures}

Figure 1.1 The Fracturing Process 2

Figure 2.1 Different Fluidized Bed Setups Used in Coating 16

Figure 2.2 Expansion Chambers for Wurster Columns 20

$\begin{array}{lll}\text { Figure } 3.1 & \text { Wurster Bed Design } & 24\end{array}$

Figure 3.2 Flowsheet for the Fluidized Bed 25

Figure 3.3 Press for Particle Strength Measurement 31

Figure $3.4 \%$ breakage of Nu-Pareil particles at different loading in a bed of sand 34

Figure 4.1 Test Results for 2 Different Levels of Polyurethane Coating 38

Figure 4.2 AP released after 10 min. as a Function of \% Coating of Acrylate 40

Figure 4.3 Size Distribution for Coating Runs with 15\% Acrylate 42

Figure 4.4 Release Rates for Different Size Distributions of 15\% Acrylate 43

Figure 4.5 Release for Different Particle Sizes Produced in the Same Batch 45

Figure 4.6 Effect of Curing Temperatures on Release for 10\% Acrylate Coating 47

Figure $4.7 \%$ AP Released after $10 \mathrm{~min}$ as a Function of \% Silica in the Coat 49

Figure $4.8 \%$ Release for Agglomerates coated with 25\% acrylate and 60\% silica 52

Figure 4.9 \% AP Leached for Longer Time Periods of Coated Agglomerates 53

Figure 4.10 Performance of Agglomerates Compared to Standard Breakers 55

Figure 4.11 Agglomerate with 25\% Acrylate Coating 57

Figure 4.12 SEM Image of Sliced Agglomerate with $25 \%$ Acrylate Coating 58

Figure 4.13 Crushed Agglomerate with $25 \%$ Acrylate Coating 59

Figure 4.14 Mechanism of Formation and Breakage of Agglomerates 60

Figure 4.15 \% AP released as a Function of \% Crosslinker in the Coat 62 


\section{List of Tables}

Table 2.1 Comparison of Three Fluid-Bed Coating Processes 17

Table 3.1 Operating Conditions in the Fluidized Bed 29 


\section{Chapter 1}

\section{INTRODUCTION}

In the oil and gas production industry, it is common practice to rupture subterranean formations in order to increase their gross permeability or conductivity. These processes are identified generally as fracturing processes. For example, it is a conventional practice to hydraulically fracture a well in order to produce one or more cracks or "fractures" in the surrounding formation by the mechanical breakdown of the formation. Fracturing may be carried out in wells, which are completed in subterranean formations for virtually any purpose. The usual sites for fracturing, or other stimulation procedures, are production wells having oil and/or gas reserves. Injection wells are used in secondary or tertiary recovery operations, wherein the injection of fluids is accomplished after fracturing the subterranean formations. Hydraulic fracturing is accomplished by injecting a hydraulic fracturing fluid into the well and applying sufficient pressure on the fracturing fluid to cause the formation to rupture with the attendant production fractures. This is shown in Figure 1.1.

Fracturing fluids, often, contain a variety of materials such as sand and other additives to hold open the fracture. In order to prevent these solids from settling out before they reach the fracture, it is common practice to prepare the fracturing fluid as a very high viscosity liquid or gel. After the high viscosity fracturing fluid has been pumped into the formation and fracturing has occurred, it is desirable to remove the fluid from the formation to stimulate hydrocarbon flow through the new fractures. Generally, the removal of the highly viscous fracturing fluid is realized by "breaking" the gel or emulsion or, in other words, by converting the fracturing fluid into a low viscosity fluid. Breaking the gelled or emulsified fracturing fluid has commonly been accomplished by treating with a "breaker", 


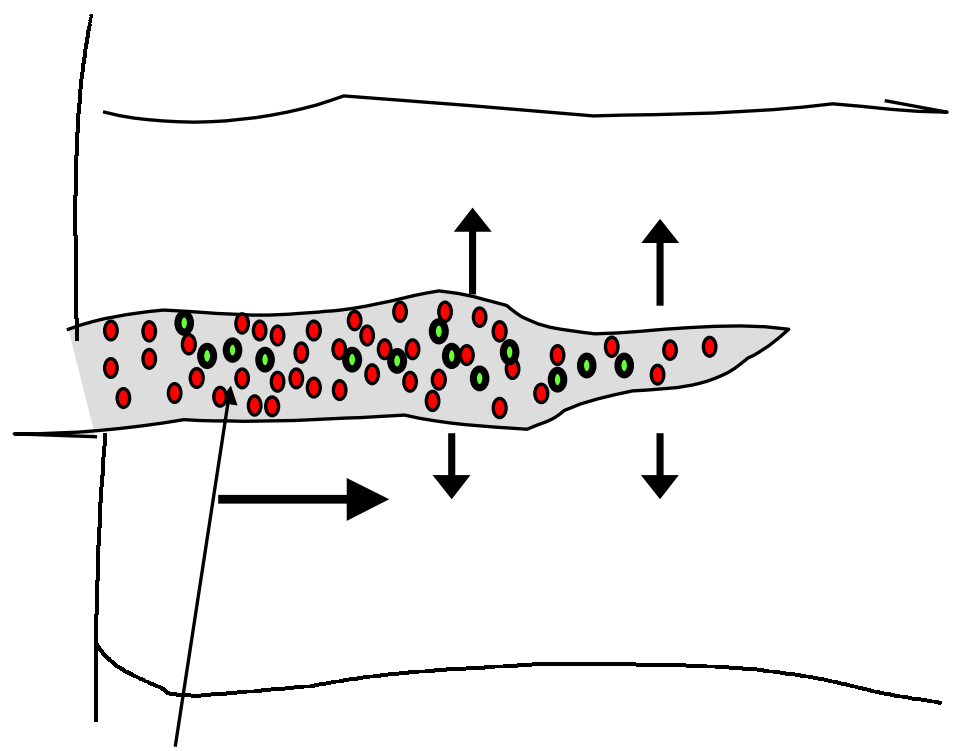

Fracturing Fluid

(a)

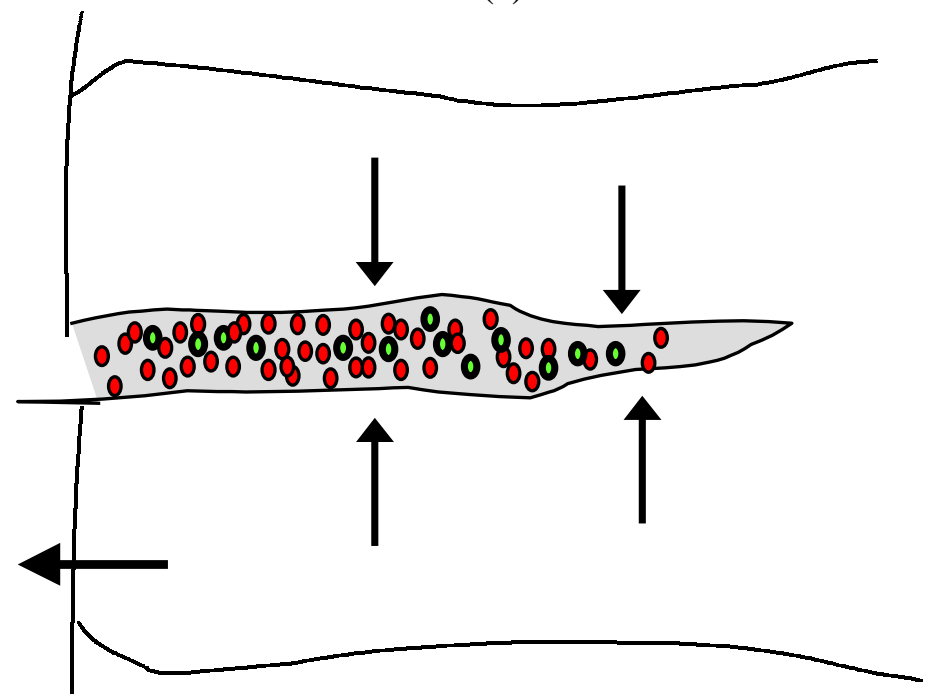

(b)

o Proppant

- Breaker particles

Figure 1.1: Schematic Diagram of the Fracturing Process 
that is, a viscosity-reducing agent. The viscosity-reduction should occur only after the fracture of the subterranean formation has taken place.

The aim of this research was to evaluate the effectiveness of coating breaker particles, which are relatively weak, with a hard brittle coating. The purpose of this coating was to provide a hard and impermeable moisture barrier for the particles that would suffer brittle fracture when exposed to a large applied load or stress. The applied stress required to fracture a coated particle would be a function of the particle size, the coating thickness, and coating material properties. The properties of the coating material should include the ability to provide an effective moisture barrier and the ability to produce a strong but brittle coat. Polyurethanes and acrylates were investigated for this purpose. Emphasis was given to the acrylate coatings. 


\section{Chapter 2}

\section{LITERATURE REVIEW}

\subsection{INTRODUCTION}

Hydraulic fracturing is achieved by means of fracturing fluids, which break down the formation. The efficiency of fracturing depends on the type of fracturing fluid used, and the method used for fracturing, both of which are discussed below. Also, a description of the fracturing process is given.

Breaker particles are used as viscosity-reducing agents for the fracturing fluid during the fracturing process. A brief discussion on the selection of the breaker particles is given below. In this research, these particles were coated in fluidized beds. The different fluidized bed setups used in coating operations are discussed, along with a brief description of the coatings investigated.

\subsection{THE FRACTURING PROCESS [1]}

The hydraulic fracturing fluid is injected into the fluid well and sufficient pressure is applied on the fluid to cause the formation to rupture with the production of one or more fractures. The fracture or fractures may be horizontal or vertical and the tendency towards vertical fracture orientation increases with the increase in depth of the formation. During the fracture formation, a gel, emulsion or foam, along with a proppant (particulate matter) such as sand, is introduced into the fracture. The proppant is deposited in the fracture and functions to hold the fracture open after the pressure is released and the fracturing fluid flows back into the well. The fracturing fluid has a sufficiently high viscosity to retain the proppant in suspension or at least to reduce the tendency of the proppant to settle out of the 
fracturing fluid as it flows along the created fracture. Generally, a gelation agent and/or an emulsifier are used to provide the high viscosity to the fracturing fluid, which is needed to obtain the maximum benefits from the fracturing process.

After the high viscosity fracturing fluid has been pumped into the formation and the fracture has occurred, the fluid must be removed from the formation to allow hydrocarbon production through the new fractures. Generally, the removal is achieved by adding a "breaker", that is, a viscosity-reducing agent, to the fracturing fluid prior to pumping into a subterranean formation. The breaker breaks the gelled or emulsified fracturing fluid, converting the fracturing fluid into a low viscosity fluid. This helps in removing the fluid from the formation. However, this technique can be unreliable and sometimes results in incomplete breaking of the fluid and/or premature breaking of the fluid before the fracturing process is complete. Premature breaking can decrease the length of fractures obtained. Further, it is known that most fracturing fluids will break with increase in time and temperature. However, economics demands that the well be returned back to production as quickly as possible. Therefore, a good method for breaking the fracturing fluid will be one that will cause the maximum viscosity reduction in the fracturing fluid in the shortest possible time after the fracture has occurred.

\subsection{FRACTURING FLUIDS [2]}

Fracturing fluids are pumped into underground formations to stimulate oil and gas production. To achieve successful stimulation, the fracturing fluid must have certain physical and chemical properties. The fluid should be compatible with the formation material and the formation fluids. It should have the capacity to suspend proppants and to 
transport them deep into the fracture. It should be capable of developing the necessary fracture width. It should have low fluid loss, and should be easy to remove from the formation. It should have low friction pressure. It should be simple and easy to prepare the fluid in the field. It should be stable throughout the treatment. In addition, the fluid should be cost-effective.

Compatibility is a critical property of the fracturing fluid. The chemical nature of the fracturing fluid must not cause swelling of naturally occurring clays in the formation, as this might plug the pore channels. The fluid should not cause migration of fines and/or clays in the formation. The fluid must not create emulsions and/or sludging of the crude oil, which might cause plugging rather than stimulation. The fluid should not dissolve the cementing material that holds the grains of sandstone together, as this might result in spalling of the formation. The fluid must not cause scaling or paraffin problems.

Another important characteristic of the fracturing fluid is that it should be capable of transporting the proppants through the perforations and deep into the fracture. Depending on the nature of the fracturing fluid, it may perfectly suspend the proppant or, as in the case of linear fluids, allow for some settling and banking of the proppant in the fracture. It should be capable, through its inherent viscosity, to develop the necessary fracture width to accept proppants or to allow deep penetration. Insufficient viscosity will not allow proppants to be transported very far into the fracture.

The fracturing fluid should be an efficient fluid. A high percentage of the fluid should remain in the fracture and not be lost to the formation. Combining high fluid viscosity with fluid-loss additives normally attains the desired fluid efficiency.

The fracturing fluid should be capable of reverting from high viscosity to low 
viscosity upon treatment with a viscosity-reducing agent. Viscosity reduction is necessary so that the treating fluid can be easily removed from the formation. High fluid viscosity in the fracture or in the formation near the fracture can reduce hydrocarbon production.

The fracturing fluid should have low friction pressure. Most modern fracturing fluids will pump at pressures lower than low-viscosity base fluids, such as water or oil, through turbulence suppression by long-chain polymer systems. If a fluid cannot be pumped easily, normally it is not acceptable as a fracturing fluid.

The fracturing fluid should be stable so that it will retain its viscosity throughout the treatment. This is a critical aspect of any fluid. A fluid that rapidly loses its viscosity because of thermal thinning or degradation is not applicable for treatment of hightemperature wells.

Finally, the fracturing fluid should be cost-effective and easy to mix in the field. Cost-effectiveness is the most important and realistic selection criterion for a fracturing fluid. Obviously, a fluid that will not yield cost-effective stimulation will not be an acceptable fluid.

Water-based fracturing fluids are used in the majority of hydraulic fracturing treatments today. Guar gum is added to the water as a viscosifier. Guar polymer comes from a bean that when added to water thickens and viscosifies the fluid.

The particles coated in this research are suitable for a fracturing fluid, which is water-based and consists of $2 \%$ potassium chloride and $0.2-0.5 \%$ guar gum. The fluid is very viscous in nature, and has a $\mathrm{pH}$ of approximately 11. 


\subsection{METHODS FOR RELEASING FRACTURING FLUID BREAKERS}

Gel breakers are used to reduce the viscosity of polymeric fracturing fluids. It should be noted that most fracturing fluids are broken by direct addition of a viscosity breaker. However, the controlled release of the breaker has clear advantages. A variety of external means - including those dependent on chemical reactions, time/temperature processes, and subterranean process - could be used to release the breaker. While numerous processes have been described in the literature, only the more important processes are discussed below.

First, a chemical may be released into an aqueous fluid by combining the chemical with a solid hydratable gelling agent and a breaker for the gel formed by the gelling agent when hydrated [3]. The mixture is formed into prills or pellets, preferably having a size and range of from about 20 to about 40 mesh (U.S. Sieve Series), which corresponds to particle diameters from about 425 to about $850 \mu \mathrm{m}$. By combining the pellets with an aqueous fluid into which the chemical is released, the gelling agent in the pellets hydrates. Thus, it forms a protective gel around each of the pellets for the time period required for the protective gel to be broken by the gel breaker in the pellets. Once the gel breaker has broken the protective gel, the chemical in the pellets is released into the aqueous fluid. The time required for the protective gel to be broken is varied by varying the quantities of hydratable gelling agent and the gel breaker utilized in the pellets and by using different gelling agents and gel breakers.

Injecting a capsule comprising an enclosure member containing the breaker, into the subterranean formation [4] can also break the fracturing fluid. The enclosure member is sufficiently permeable to at least one fluid existing in the subterranean environment or injected with the capsule such that the enclosure member is capable of rupturing upon 
sufficient exposure to the fluid, thereby releasing the breaker. Pressure is generated within the enclosure member due to the fluid penetrating into the capsule. The increased pressure causes the capsule to rupture, releasing the breaker. This method for release of the breaker would result in the release of substantially the total amount of breaker contained in the capsule at one particular point in time. Alternately, the enclosure member may be capable of dissolving or eroding off upon sufficient exposure to the fluid, thereby releasing the breaker [5].

A percarbonate, perchlorate or persulfate breaker encapsulated within a polyamide layer can also break the fluid [6]. The polyamide membrane is permeable to at least one fluid in the formation, which dissolves the breaker, and the breaker then diffuses through the membrane to break the fracturing fluid with the membrane staying intact during the breaker release.

Finally, controlled breaking of an aqueous based fracturing fluid [2], can be achieved by using an encapsulated breaker, which is capable of providing a controlled release at elevated $\mathrm{pH}$ in aqueous-based fracturing fluids. The encapsulated breaker is enclosed within an inert membrane that is permeable to at least one fluid present in a subterranean formation or to a carrier fluid introduced into a subterranean formation with the encapsulated breaker. With this technology, the fluid permeates the encapsulated breaker and causes the breaker to diffuse through voids in the membrane and into the fracturing fluid. The membrane stays substantially intact during the period of release of the breaker, thereby providing controlled release. This provides an encapsulation membrane which is capable of functioning in an aqueous based fluid at temperatures of from $60^{\circ} \mathrm{F}$ to about $300^{\circ} \mathrm{F}$ and at a fluid $\mathrm{pH}$ of up to 12 without premature release of the 
breaker into the fluid. In addition, as the load water is returned to the wellbore upon completion of the treatment, the breaker capsules can continue to release breaker into any filter cake which is present, thus assisting in dissolving and removing the filter cake from the formation.

During the fracturing treatment, the proppant is subjected to high hydrostatic pressure. After completion of the treatment, the pressure dissipates through fluid loss and the fracture closes. Within the proppant pack, point-to-point stresses are very high. Use of the closure process to trigger the breaker release is very attractive because it occurs automatically after the end of the treatment. Nolte [7] has suggested that introducing a viscosity-reducing chemical contained within hollow or porous, crushable and fragile beads along with the fracturing fluid may reduce the viscosity of the fracturing fluid introduced into a subterranean formation. When the fracturing fluid passes or leaks off into the formation or the fluid is removed by back flowing, any resulting fractures in the subterranean formation close and crush the beads. The crushing of the beads then releases the viscosity-reducing chemical into the fluid. This process is dependent on the closure pressure of the formation to obtain the release of the breaker and thus depends upon the formation and its closure rate.

The particles that were coated in this research would be used in a fracturing fluid. These particles take advantage of the stress within the fracture as the primary release mechanism (as described above). Due to the pressure generated by the closure of the formation, the coating would break and the breaker chemicals would be released. Hence, the objective of this research was to develop a coating, that would provide a high moisture barrier for the particles, but would undergo brittle fracture when exposed to high pressures. 
The delayed release breaker would be released in localized sites. To maximize the release, the breaker size must be larger than the pore size of the sandpack. Larger particles (lower total surface area) favor minimal premature release; however, smaller particles (larger number of particles) maximize dispersion within the pack. Overall, a breaker that is roughly the same size or slightly larger than the proppant would be desirable.

\subsection{THE ENCAPSULATED BREAKER [8]}

An ideal breaker should have the following properties:

1. It should have minimal effect on proppant transport or fluid loss during the fracturing treatment.

2. It should remain in the fracture (should not be removed from the fracture through fluid loss)

3. The breaker should minimize proppant-pack permeability damage.

4. It should degrade the fracturing fluid rapidly after the treatment.

5. It must be stable during storage.

Sequestering the breaker by isolating it as a separate, dispersed phase within the fluid would meet most of the criteria. Premature fluid degradation can be avoided by allowing the breaker to react with the fluid only after the fracturing has taken place.

The breaker, if a solid, must also be sufficiently durable to withstand the mixing, pressure, and pumping in field equipment. It must also survive transport at high rates through the tubing, and then long residence times in long, narrow, rough fractures. The breaker must be capable of being mixed easily with fluids. It must have a long shelf life under highly variable storage conditions and should require minimal preparation before use. 
High concentrations of the breaker are required to reduce significant proppant-pack permeability damage. The core material must effectively degrade guar-based viscosifiers at a reasonable concentration. To moderate the quantities required, maximizing the breaker content within a capsule is advantageous. However, many oxidizing agents become increasingly unstable upon concentration and may also become less stable when adsorbed on a solid substrate. Fortunately, some strong oxidizing agents are available in a solid, concentrated form that becomes active when in water.

Persulfate salts (i.e., peroxydisulfate) are available in their crystalline forms. These salts are among the strongest oxidizing agents known and are widely used commercially as (dissolved) fracturing-fluid breakers where they rapidly reduce viscosity. Persulfates also have another advantageous property: during the reaction with the guar (or aqueous decomposition), 2 mole of sulfuric acid (a strong acid) can be generated per 1 mole of persulfate. A dual-mode oxidative/acidic degradation of guar-based viscosifiers is possible if the acid concentration becomes sufficiently high. Ammonium Persulfate (AP) is stable in the dry form to at least $220^{\circ} \mathrm{F}$. AP was selected for the oxidant core with a 20/40-mesh size to match the most commonly used proppant size.

The characteristics of the coating material are very important in this research. A review of some of the common types of coating is given in the next section.

\subsection{COATING FORMULATION}

\subsubsection{Aqueous vs. Organic Coating:}

A number of reasons exist for the present interest in aqueous coating systems (Pondell [9]). The cost of organic solvents has dramatically increased in the past ten to 
fifteen years and the government has introduced new regulations concerning these solvents. The Environmental Protection Agency (EPA) has become increasingly stringent on solvent and solvent-vapor discharge to the environment. This poses economic problems because solvent and solvent-vapor recovery systems tend to be very expensive.

Organic solvents are generally explosive or highly flammable. This has led to severe limitations in the use of certain solvents. The Occupational Safety and Health Agency (OSHA) tightened worker exposure to solvent vapors, thereby greatly increasing the requirements for better ventilation. These factors have induced many companies to switch to water as the coating solvent.

\subsubsection{Coating Compositions}

The reactive core must be tightly sequestered to prevent viscosity reduction until the release is triggered [8]. Ammonium Persulfate (AP) rapidly dissolves and becomes highly reactive when it contacts water. A very effective water barrier is required to maximize sequestration, yet the barrier must be amenable to release by applied stress. Processing issues must also be addressed.

\subsubsection{Polyurethane Coatings}

Two-component polyurethanes are particularly suited for high-solids coatings because they are comprised of lower molecular weight oligomers, which react on the substrate to form a polymeric network [10]. The reaction of an isocyanate with a hydroxyl-containing compound (Equation 2.1) is the basis for polyurethane formation. 


$$
\mathrm{R}-\mathrm{N}=\mathrm{C}=\mathrm{O}+\mathrm{R}^{\prime}-\mathrm{OH} \rightarrow \mathrm{R}-\mathrm{NH}-\mathrm{C}-\mathrm{O}-\mathrm{R}^{\prime}
$$

Polyurethane coatings exhibit outstanding performance properties, such as hardness, and solvent and abrasion resistance, allowing them to be used for many different applications. The disadvantage of polyurethane coatings is that they are flexible in nature. A route to produce resistant and brittle coatings based on the urethane chemistry was investigated in this work.

\subsubsection{Acrylate coatings}

A chemical formulation for acrylate coatings is given by Norman and Laramay [1]. They suggest that the coating for the breaker particle should comprise of a partially hydrolyzed acrylic, preferably in an aqueous based form, which is crosslinked with either an aziridine prepolymer or a carbodiimide.

The partially hydrolyzed acrylic is any of the vinyl acrylic latex polymers containing from about $0-60 \%$ by weight monovinyl aromatic content of styrene, from about $5-25 \%$ by weight alpha, beta unsaturated carboxylic acid content and from about $15-95 \%$ by weight alkyl acrylate or methacrylate ester content.

The aziridine prepolymer can comprise, for example, pentaerythritol-tris- $[\beta-$ (aziridinly) propionate]. The carbodiimide can comprise, for example, 1,3dicyclohexylcarbodiimide. The cross-linking agent used in this work was an aziridine prepolymer, commercially available from Sybron Chemicals (Ionac, Ion Exchange Resin, PFAZ-322). 
Discussions with Norman [11] suggested that by utilizing the above chemistry and by adding some inert filler in the coat (such as silica), a brittle coat might be achieved.

\subsection{THE COATING PROCESS}

Selection of an encapsulation technique depends on the physical properties of the materials to be used. Most of the particle coating in this work was carried out in a fluidized bed using a bottom spray with a draft tube (insert). A brief review of this method of coating is given in the following sections:

\subsubsection{The Fluid-Bed Equipment}

Fluid-bed equipment is well known for its drying efficiency, having been used for drying and granulating for many years. The use of fluid-bed equipment in applying aqueous coating systems has increased greatly due to improved drying efficiency, and improved design considerations over previous coating systems.

Aqueous film coating can be applied to the fluidized material by a variety of techniques, including spraying from the top (granulator or conventional mode), from the bottom (Wurster), or tangentially (rotary granulator). (Figure 2.1 from Mehta [12]).

The advantages, disadvantages and applicability of each type are shown in Table

\section{1.}



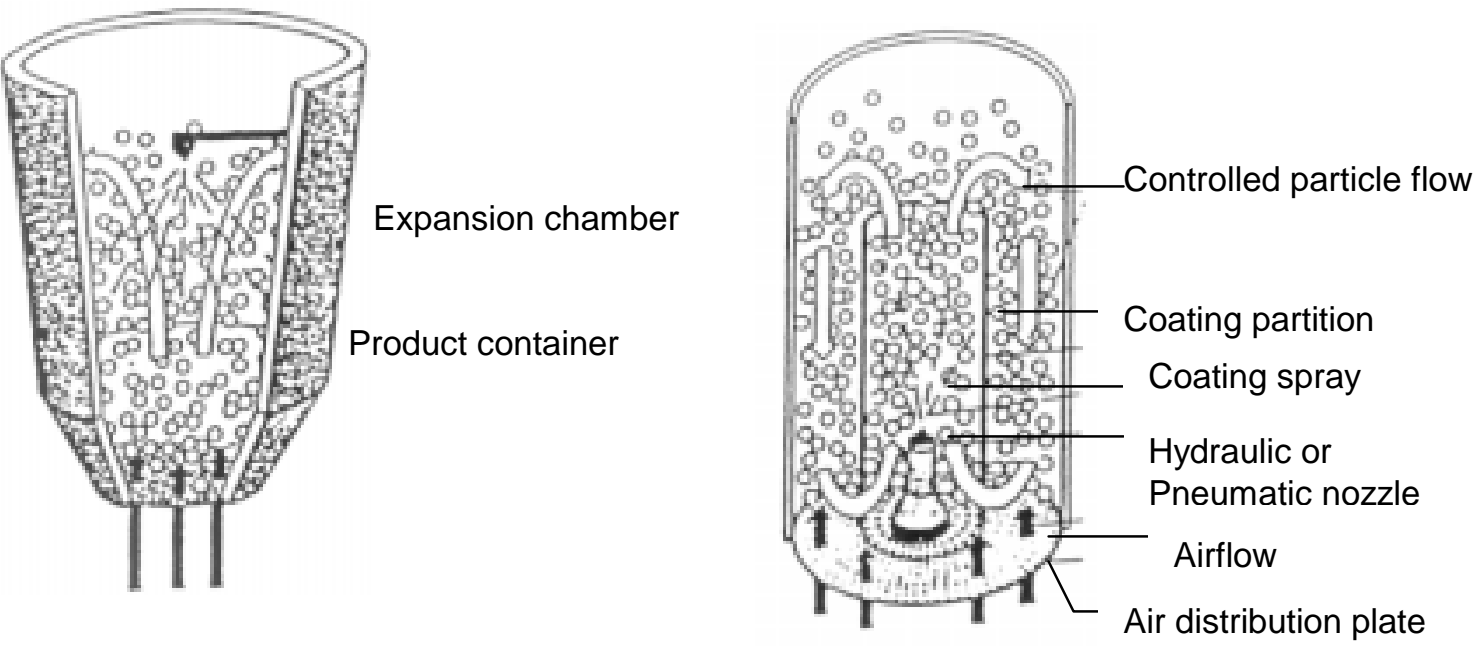

TOP SPRAY METHOD USED IN CONVENTIONAL GRANULATION COATERS

\section{BOTTOM-SPRAY METHOD USED IN WURSTER AIR-SUSPENSION COLUMNS}

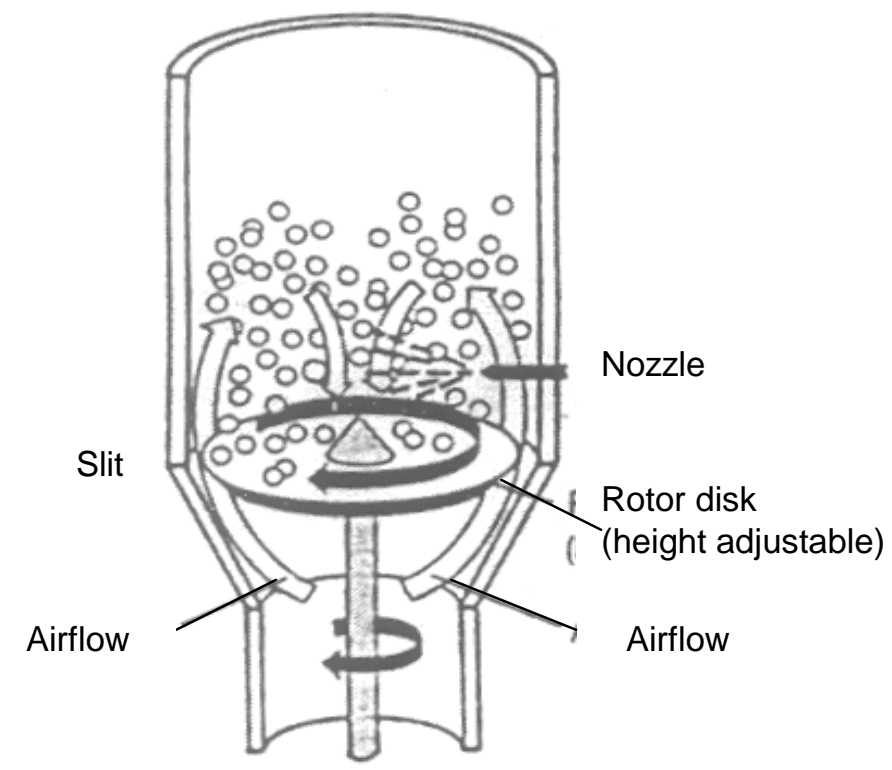

TANGENTIAL-SPRAY METHOD USED IN ROTARY FLUID-BED COATERS

Figure 2.1: Different Fluidized Bed Setups Used in Coating [12] 
Table 2.1: Comparison of Three Fluid-Bed Coating Processes (Mehta [12])

\begin{tabular}{|c|c|c|c|}
\hline Processing Method & Advantages & Disadvantages & Applications \\
\hline $\begin{array}{l}\text { Top-spray coating } \\
\text { (conventional mode) }\end{array}$ & $\begin{array}{l}\text { Accommodates large } \\
\text { batch sizes, is simple } \\
\text { to set up, and allows } \\
\text { easy access to nozzle }\end{array}$ & $\begin{array}{l}\text { Limited in its } \\
\text { applications }\end{array}$ & $\begin{array}{l}\text { Hotmelt coating and } \\
\text { aqueous enteric } \\
\text { coatings } \\
\text { Not recommended } \\
\text { for sustained-release } \\
\text { products }\end{array}$ \\
\hline $\begin{array}{l}\text { Bottom-spray } \\
\text { coating (Wurster) }\end{array}$ & $\begin{array}{l}\text { Accommodates } \\
\text { moderate batch } \\
\text { sizes, produces } \\
\text { uniform and } \\
\text { reproducible film } \\
\text { characteristics, and } \\
\text { allows for widest } \\
\text { application range }\end{array}$ & $\begin{array}{l}\text { Tedious to set up, } \\
\text { does not allow } \\
\text { access to nozzles } \\
\text { during processing, } \\
\text { and is the tallest } \\
\text { fluid-bed machine } \\
\text { for coating fine } \\
\text { particles }\end{array}$ & $\begin{array}{l}\text { Sustained-release, } \\
\text { enteric-release, and } \\
\text { layering }\end{array}$ \\
\hline $\begin{array}{l}\text { Tangential-spray } \\
\text { coating (rotary } \\
\text { mode) }\end{array}$ & $\begin{array}{l}\text { Simple to set up, } \\
\text { allows access to the } \\
\text { nozzle during } \\
\text { processing, permits } \\
\text { higher spray rates, } \\
\text { and is the shortest } \\
\text { fluid-bed machine } \\
\text { for coating fine } \\
\text { particles }\end{array}$ & $\begin{array}{l}\text { Puts mechanical } \\
\text { stress on the product }\end{array}$ & $\begin{array}{l}\text { Very good for } \\
\text { layering, sustained- } \\
\text { release, and enteric- } \\
\text { coated products } \\
\text { Hotmelt coating } \\
\text { possible } \\
\text { Not recommended } \\
\text { for friable products }\end{array}$ \\
\hline
\end{tabular}




\subsubsection{Bottom Spray (Wurster)}

The Wurster coating system (Mehta [13]) was patented almost 40 years ago. It has had considerable success in particle coating in the pharmaceutical and agricultural industries.

The "Wurster" process is a multipass coating technique. The essential part of this process is the design of the coating chamber. A bed of particles is fluidized by a column of heated air drawn into the chamber from below the distribution plate. The Wurster partition (draft tube) and the distribution plate control the airflow and hence the aeration of solids in the bed. The pattern and size of holes in the plate are such that a majority of the air is diverted through the draft tube, causing fluidization and upward travel of the particles. As the particles exit the partition and enter an expansion zone, air velocity decreases and the particles disengage and settle in the annular region between the partition and the outer bed wall. The air in this downward moving bed acts to cushion the particles as they travel downward to continue their cycling through the coating zone. The balance between the air inside and that outside the partition and the gap between the distribution plate and the partition are critical. Liquid application rates may be quite high. Additionally, the disengaging height (the distance the particles rise above the partition) in such equipment is small and is the key to minimizing the attrition that is usually associated with airsuspension particle coating. As the particles cycle through the partition, the spray nozzle mounted below the partition applies a coating. The desired coating thickness is reached after many passes. The incoming heated air not only fluidizes the bed but also dries the sprayed particles. 
Successful production of encapsulated particles is controlled by many operating parameters in the Wurster method. The Wurster system is growing in popularity in the coating of smaller particles. Using such a device, it is possible to apply droplets to the substrate before much evaporation occurs and to rapidly evaporate surface solvent once coating has taken place. Discretely dividing the particles by air suspension allows the application of films to pellets, granules, and materials as fine as $50 \mu \mathrm{m}$ with little or no agglomeration (depending on the coating substance). The organization of the particles in close proximity to the liquid nozzle and rapid bed cycle times yield uniform distribution of the film. There exist many variations in the geometry of the fluid bed system. However, it is recommended that longer expansion chambers be used to coat small particles as shown in Figure 2.2. 


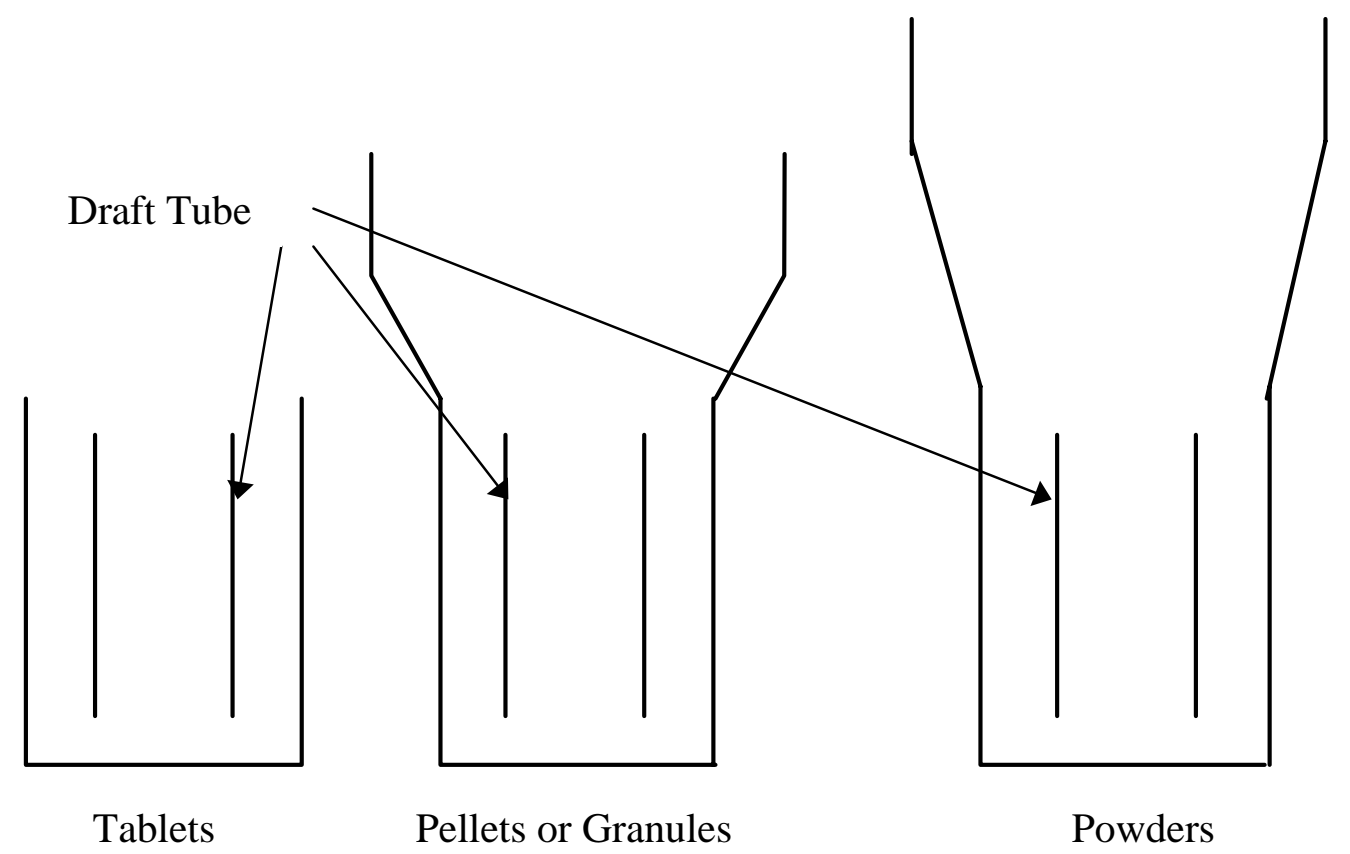

Figure 2.2: Expansion Chambers for Wurster Columns [13] 


\section{Chapter 3}

\section{EXPERIMENTAL EQUIPMENT AND PROCEDURES}

\subsection{EXPERIMENTAL METHODOLOGY}

The methodology that was followed in this research consisted of the three stages given below.

- Coating Formulations: Investigation of coating compositions that would provide brittle coatings for particles, and which were aqueous based.

- Coating Process: Investigation of the variables for the process of coating particles with different chemicals, satisfying the above requirements.

- Strength Tests on Coated Particles: Investigation of the strength, water resistance, and susceptibility to brittle fracture of the particles. Simple test methods were developed to compare samples from different coating runs.

\subsection{COATING FORMULATION}

The main objective for the selection of the coatings was the fulfillment of the following requirements:

- It should be strong but brittle.

- It should be able to provide an impermeable moisture barrier to the particles that would suffer brittle fracture upon compression.

- It should be easily coated.

- It should be aqueous based.

During this work, coatings belonging to the following 2 groups were investigated:

- Polyurethane coatings 
- Acrylate coatings

\subsubsection{Polyurethane Coatings}

High molecular weight isocyanates, in combination with hydroxyl-containing compounds, are believed to form hard and moisture-resistant polyurethane coatings. Diphenyl Methane Diisocyanate (MDI) is the aromatic polyisocyanate which is produced worldwide in the largest volume [14]. MDI is commercially available from Bayer Co., as MONDUR MR.

The following polyurethane system was investigated:

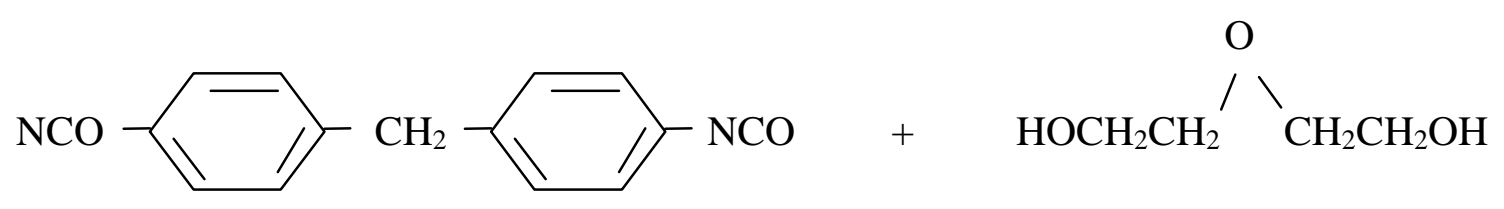

Diphenyl Methane Diisocyanate (MDI)

Diethylene Glycol (DEG).

This product offers important features such as low viscosity, high reactivity, and economy.

\subsubsection{Acrylate Coatings}

A proprietary acrylic dispersion available form Halliburton was used for the coating purposes.

An aziridine prepolymer, PFAZ-322, commercially available from Sybron Chemicals, was added to the coating solution as a cross-linking agent. The amount added was $3 \%$ by weight of the coating.

The effect of the addition of silica to the acrylate was also investigated. The 
acrylic was mixed with particulate micron sized silica prior to coating of the breaker. This formulation is based on a patent by Halliburton [1]. The addition of inert particles, like silica, to the partially hydrolyzed acrylic coating creates imperfections in the coating. The purpose of the silica, according to the patent, is to increase the permeability of the coat and allow diffusion of the breaker chemicals into the fracturing fluid. However, it was also suggested, through discussions with Norman and Turton [11], that the addition of a larger amount of silica could increase significantly the brittleness of the coating. This effect was investigated in detail, and the results are presented later.

\subsection{COATING PROCESS}

\subsubsection{Polyurethane Coatings}

The procedure used to coat particles with a polyurethane layer consisted of adding $1.5 \mathrm{~g}$ of MDI liquid to $100 \mathrm{~g}$ of particles contained in a glass bottle. The mixture was shaken so as to uniformly coat the particles with the MDI. After one minute of shaking, $0.6 \mathrm{~g}$ of DEG was added to the mixture and shaken for a further five minutes. This procedure essentially deposited a $2 \mathrm{wt} \%$ coating on the surface of the particles. This coating was allowed to cure for a period of at least 4 hours. After this time, the procedure was repeated. In this way, successive layers of approximately 2 wt $\%$ of polyurethane were built up on the surface of the particles.

\subsubsection{Acrylate Coatings}

The coating of particles was carried out in a Wurster coater. The bed design is illustrated in Figure 3.1 and the flowsheet for the process is shown in Figure 3.2. 


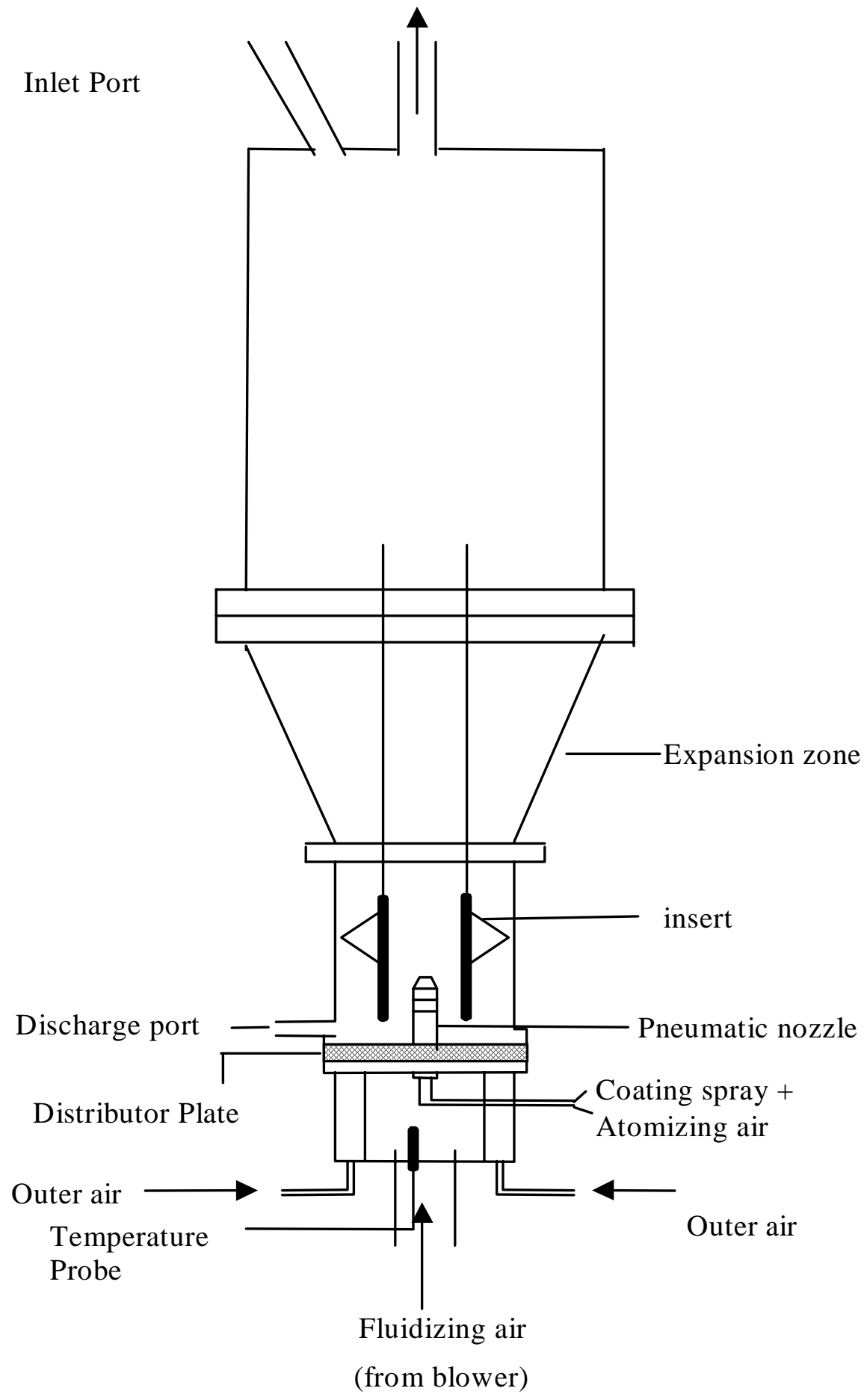

Figure 3.1: Wurster Bed Design 

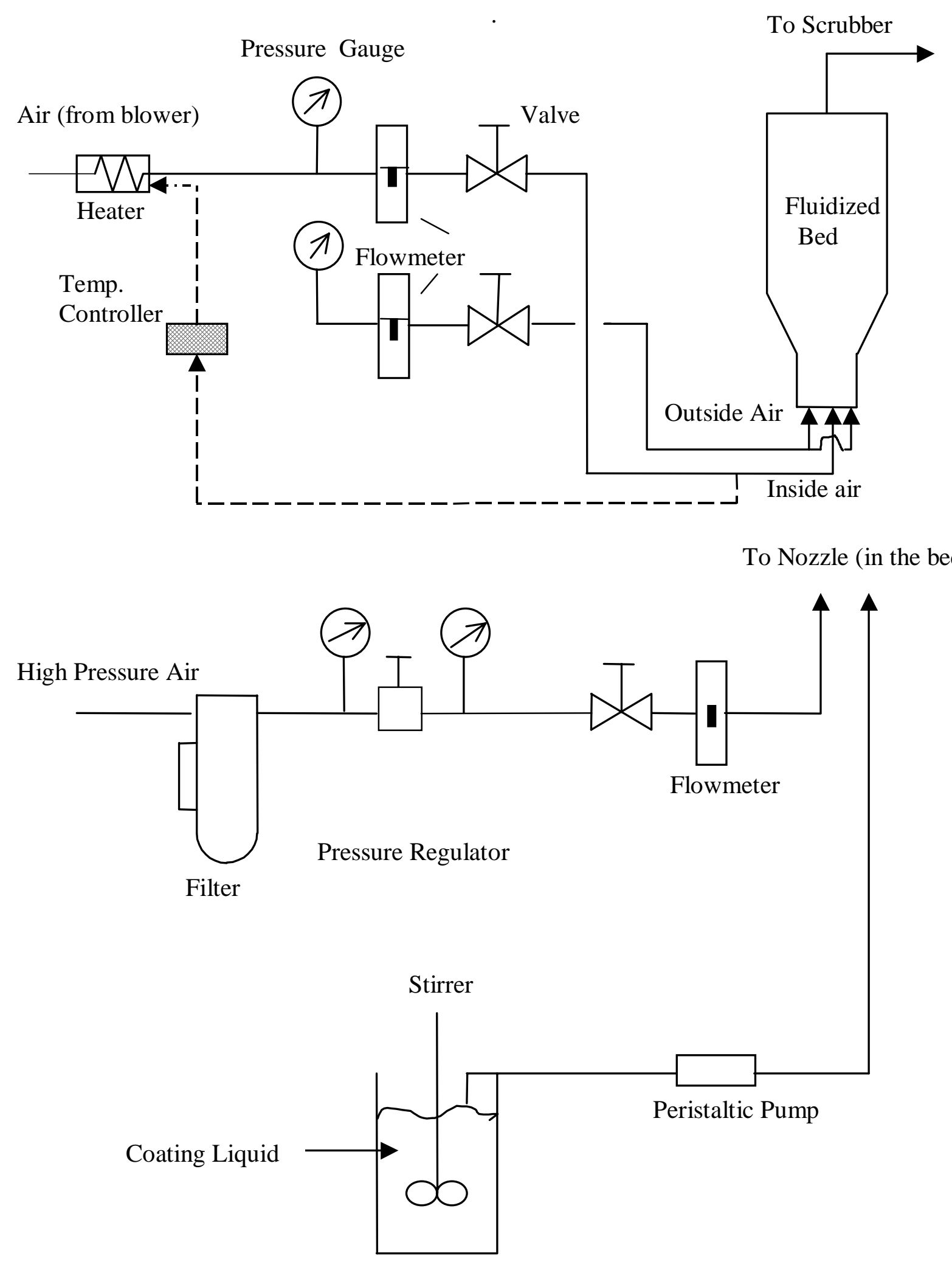

- - - - - Control Signal

Figure 3.2: Flowsheet for the Fluidized Bed 


\subsubsection{Coating Procedure}

At the beginning of the experiment, the bed was loaded with a weighed amount $(1000 \mathrm{~g})$ of particles from the input port located at the top of the bed. The blower/blowers (Ring Compressor Model \# VFC904A-7W from Fuji Electric Co.) was then turned on and the flowrates for the inside and the outside air were adjusted. Next the heater was turned on. The required temperature was obtained by adjusting the temperature controller (Antunes TCE Temperature Controller and Indicator Model \# 2408PL-04W-B40 available from A.J. Antunes \& Co.). The cooling fan on the control box was run all of the time to prevent excessive heating of the controller.

It took 10-15 min for the fluidizing air to heat the bed of particles to the required temperature. In the meantime, the atomizing air was turned on to the required pressure and flowrate. The coating liquid was continuously stirred. The coating process was started once the air was heated. This involved pumping the liquid from the stirred container via the peristaltic pump (Masterflex C/L Compact Dual-Channel Pump Model \# P-77120-60) to the nozzle placed in the bed. Depending on the size of the tygon tubing in the peristaltic pump, different flow ranges were obtained.

Once the coating was completed, the heater was turned off, and then the blowers were run for 10-15 min for the air to cool before the blowers were shut off. This was done to ensure that the heat did not conduct into the PVC lines and soften and possibly melt them. Next the coated particles were removed from the discharge port located just above the distributor plate. Some particles did not come out and had to be removed when the bed was dismantled. 


\subsubsection{Operating Conditions}

Different operating conditions were tried with the goal of achieving the optimum conditions for coating. The important operating variables were spray rate, size of spray nozzle, atomizing air pressure, fluidizing air flow within the partition (draft tube), fluidizing air flow in the annulus, partition gap height and temperature within the bed. Each of these operating parameters is discussed below.

Spray rate: At low spray rates $(<5 \mathrm{ml} / \mathrm{min})$ it was found that particles would start agglomerating after a certain time, and form large lumps within the bed until a stage was reached when fluidization would stop. A maximum coating of approximately $5 \%$ could be achieved by this time. This was due to the fact that the coating took a long time to dry. Spraying at higher spray rates $(10 \mathrm{ml} / \mathrm{min})$ was found to be a better option, as this reduced the spray time required to achieve a certain level of coating. Coating levels of $15-20 \%$ were achieved with this flow rate.

Size of spray nozzle: The size of the spray nozzle was found to be an important factor affecting the final strength of coating. Larger nozzle diameters resulted in larger droplet sizes, which in turn affected the size of agglomerates being formed. This was an important factor due to the small size (20-40 mesh) of the AP particles being coated. Two nozzle diameters $(0.61 \mathrm{~mm} \& 1.07 \mathrm{~mm})$ were used in the course of this work. Effective spraying of mixtures of acrylate and silica was achieved only with the larger nozzle. Both the nozzles were effective for coating the acrylate only. The use of the different nozzle sizes resulted in different size distributions of coated particles. These results are given later in Chapter 4.

Atomizing air pressure: Lower atomizing air pressures resulted in the formation of large 
lumps of particles within the draft tube. This was most likely due to the larger liquid droplets formed by the atomization process. Very high air pressures resulted in a backpressure within the liquid line obstructing the flow of the spraying liquid. An atomizing air pressure of 40 psi was found to be optimal.

Fluidizing air flow within the draft tube: Lower air flows again caused the formation of large lumps within the draft tube due to poor solid circulation. Very high air flows caused the particles to be thrown out through the top outlet of the bed. Air flowrates of around 25 $\mathrm{scfm}$ of air for a loading of $1 \mathrm{~kg}$ of particles, and $40 \mathrm{scfm}$ of air for $2 \mathrm{~kg}$ were found to be optimal.

Fluidizing air flow in the annulus: Lower air flows (10 scfm) caused fluidization of the particles to cease around the draft tube after some time. A maximum coating of $7 \%$ was achieved at the low flowrates. Higher air flows (15 scfm) caused bubbling of particles around the draft tube. This condition was found to be preferable as this prevented particles from sticking together and forming lumps.

Partition gap height: During the final stages of this work, it was discovered that the height of the gap between the draft tube and the distributor plate was also a critical parameter, which affected the leach rates of the coated agglomerates produced. This is discussed in detail in Section 4.2.8, where the leach rates for coating runs from 2 draft tube heights are given. However, as noted before, since this was found to be an important variable only during the latter stages of the work, an exact value of the height for the initial coating runs was not measured, though it is estimated to be in the range of $1-2 \mathrm{~cm}$. Temperature in bed: A bed temperature of $40-45^{\circ} \mathrm{C}$ was found to be suitable for the coating process. 
The coating conditions are summarized in Table 3.1

Table 3.1: Operating Conditions in the Fluidized Bed

\begin{tabular}{|l|l|}
\hline Spray rate & $10 \mathrm{ml} / \mathrm{min}$ \\
\hline Size of spray nozzle & $0.61 \mathrm{~mm} \& 1.07 \mathrm{~mm}$ \\
\hline Atomizing air pressure & $40 \mathrm{psi}$ \\
\hline Fluidizing air flow within the draft tube & $25-40 \mathrm{scfm}$ \\
\hline Fluidizing air flow in the annulus & $15 \mathrm{scfm}$ \\
\hline Partition gap height & $1-2 \mathrm{~cm}$ \\
\hline Temperature in the bed & $40-45^{\circ} \mathrm{C}$ \\
\hline
\end{tabular}




\subsection{TESTS FOR DETERMINATION OF COATING STRENGTH}

The test mixture comprised of 11g Carbo-Prop sand (20-40 mesh i.e. particle sizes between $425 \mu \mathrm{m}$ and $850 \mu \mathrm{m}$ ) and $0.11 \mathrm{~g}$ coated ammonium persulfate (AP) particles. The uncoated AP particles were also of size 20-40 mesh. In order to determine whether the selected coating fulfilled the requirements of low release when uncrushed and high release when crushed, the following two tests were carried out:

1. Leach Test (should ideally give $0 \%$ release)

2. Compression Test (should ideally give $100 \%$ release)

\subsubsection{Leach Test}

The purpose of the leach test was to estimate the dissolution-time of the coated but uncrushed particles. In order for a coating to be acceptable, a low leach rate and high $\%$ of breakage under compression are desirable. In order to estimate the amount of leach for uncrushed particles, the test mixture was kept immersed in $50 \mathrm{cc}$ water and continuously stirred. After a given time, the amount of AP released was determined using iodometric titration methods. This titration procedure is given in greater detail in Section 3.4.4.

\subsection{2 $\underline{\text { Compression Test }}$}

The apparatus for determining the strength of the coating under an applied pressure is illustrated in Figure 3.3. The test mixture was placed in a test cylinder of diameter $7.62 \mathrm{~cm} \mathrm{(3").} \mathrm{A} \mathrm{piston} \mathrm{(length} 8.9 \mathrm{~cm}$ ), which was a loose fit inside the cylinder, was placed on top of the loaded particle charge and the whole assembly was placed in a 


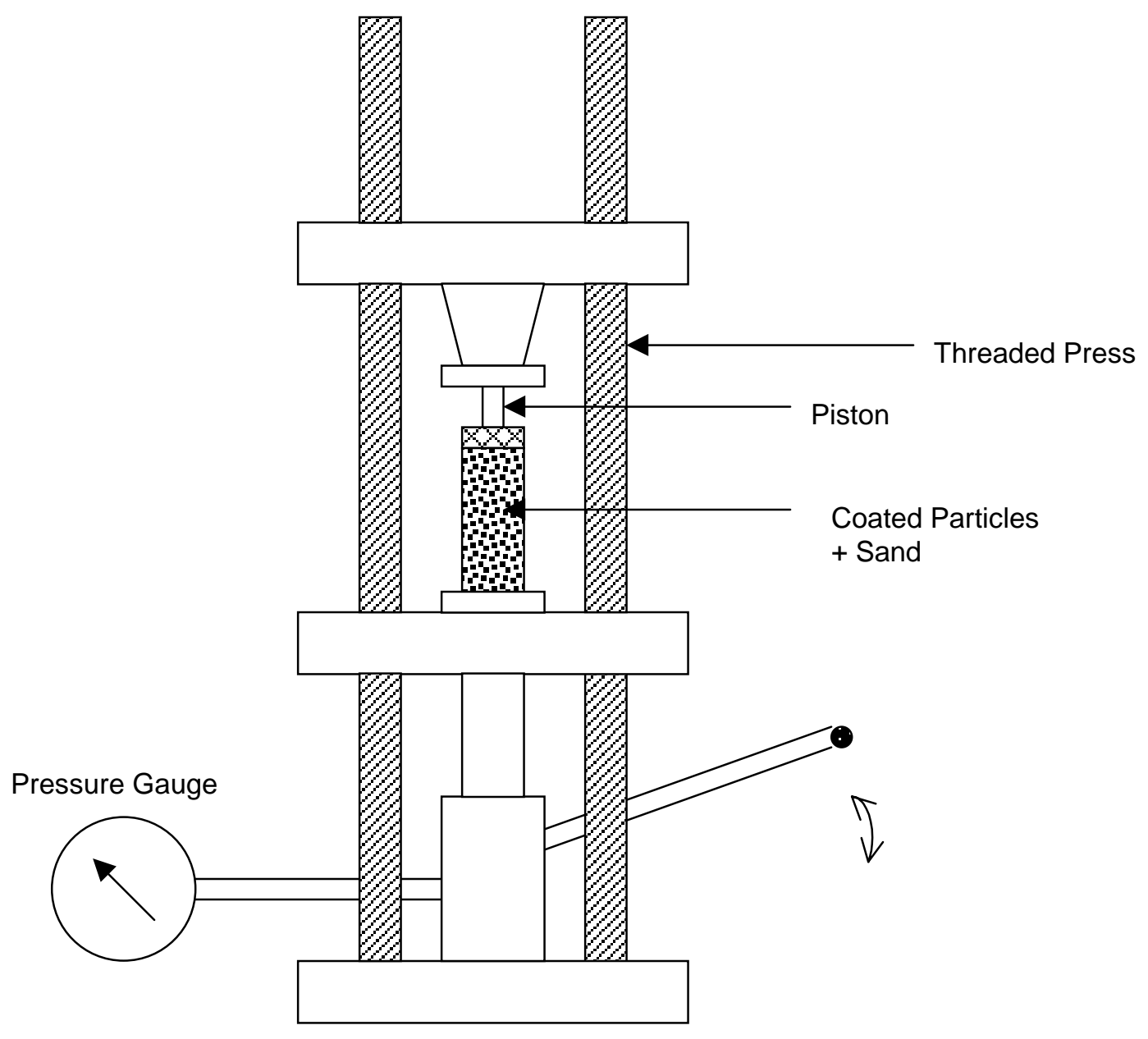

Figure 3.3: Press for Particle Strength Measurement 
hydraulic press (Carver Hydraulic Unit Model \# 3912). A load of 62,720 N (14100 lbf) was applied which corresponded to an average applied stress of $13.8 \mathrm{MPa}$ (2000psi). The load was applied over a period of one minute until a value of $62,720 \mathrm{~N}$ was reached, and then this pressure was held constant for two minutes. This procedure corresponds to API RP 56 used for testing the crush resistance test of fracture sand [15]. The fraction of broken particles was determined by measuring the concentration of AP in water using a titration procedure similar to the one given above for the leach test.

\subsubsection{Optimization of the Total Amount of Mixture to be used for the Compression}

\section{$\underline{\text { Test }}$}

In order to determine a suitable loading of the test mixture for the compression test, preliminary tests were carried out with $\mathrm{Nu}$-Pareil particles. Nu-Pareil particles are weak particles, made up of mainly sucrose. They were mixed with particulate sand, and the mixture was subjected to pressure, as described above in the procedure for the compression test. The objective of these tests was to estimate what weight of a mixture of coated particles and sand would give measurable results in the compression test. It was postulated that a loading, which gave close to $100 \%$ breakage of Nu-Pareils upon compression, would be a good loading for the tests on the coated particles. The size of the Nu-Pareil particles was 16-18 mesh (i.e. particle sizes between $1000 \mu \mathrm{m}$ and $1180 \mu \mathrm{m}$ ), while that of the sand was 20-40 mesh (i.e. particle sizes between $425 \mu \mathrm{m}$ and $850 \mu \mathrm{m}$ ). After compression, the particles were sieved to separate the particles of size greater than $1 \mathrm{~mm}$. The particles smaller than $1 \mathrm{~mm}$ corresponded to sand particles and broken $\mathrm{Nu}$ Pareil particles. The particles greater than $1 \mathrm{~mm}$ were then observed under the microscope 
to determine how many were broken. Once the number of unbroken particles in a sample was known, the total fraction of broken particles was calculated. Figure 3.4 shows the fractional breakage obtained at different loading in terms of the weight of the sample sand. The ratio of the Nu-Pareil to sand was maintained at 1:100, which is a close estimate of the ratio of the breaker particles to the proppant, during the fracturing process. The dashed lines indicate the layers of particles in the cylinder, the number of which is given in bold. It can be seen from the graph that almost $100 \%$ of breakage is obtained up to 4 layers. Above this value, the breakage drops off significantly.

All our tests were carried out using a mixture comprising of $11 \mathrm{~g}$ of sand and $0.11 \mathrm{~g}$ of coated AP particles. This corresponded to a loading of $0.24 \mathrm{~g}$ per $\mathrm{m}^{2}$ of packed sand in the compression test.

\subsubsection{Procedure for Iodometric Titration}

In order to evaluate the amount of AP leaching out of particles or released after compression, a quantitative analysis for AP was needed. The following describes the iodometric titration method used in this work. Halliburton Energy Services, Inc provided this titration method. It is a modification of an existing test for AP [18]. All the chemicals for the titration were obtained from Fisher Scientific (711 Forbes Avenue, Pittsburgh, PA 15219-4785).

Preparation of titrant sodium thiosulfate (approximately $0.0125 \mathrm{M} \mathrm{Na}_{2} \mathrm{~S}_{2} \mathrm{O}_{3}$ )

To a liter of deionized water, which was boiled and cooled to room temperature, $3.125 \mathrm{~g} \mathrm{Na}_{2} \mathrm{~S}_{2} \mathrm{O}_{3} .5 \mathrm{H}_{2} \mathrm{O}$ was added. Then 2-3 $\mathrm{g}$ of borax crystals was dissolved in it to help stabilize the solution from decomposition. In order to obtain the true molarity of the 


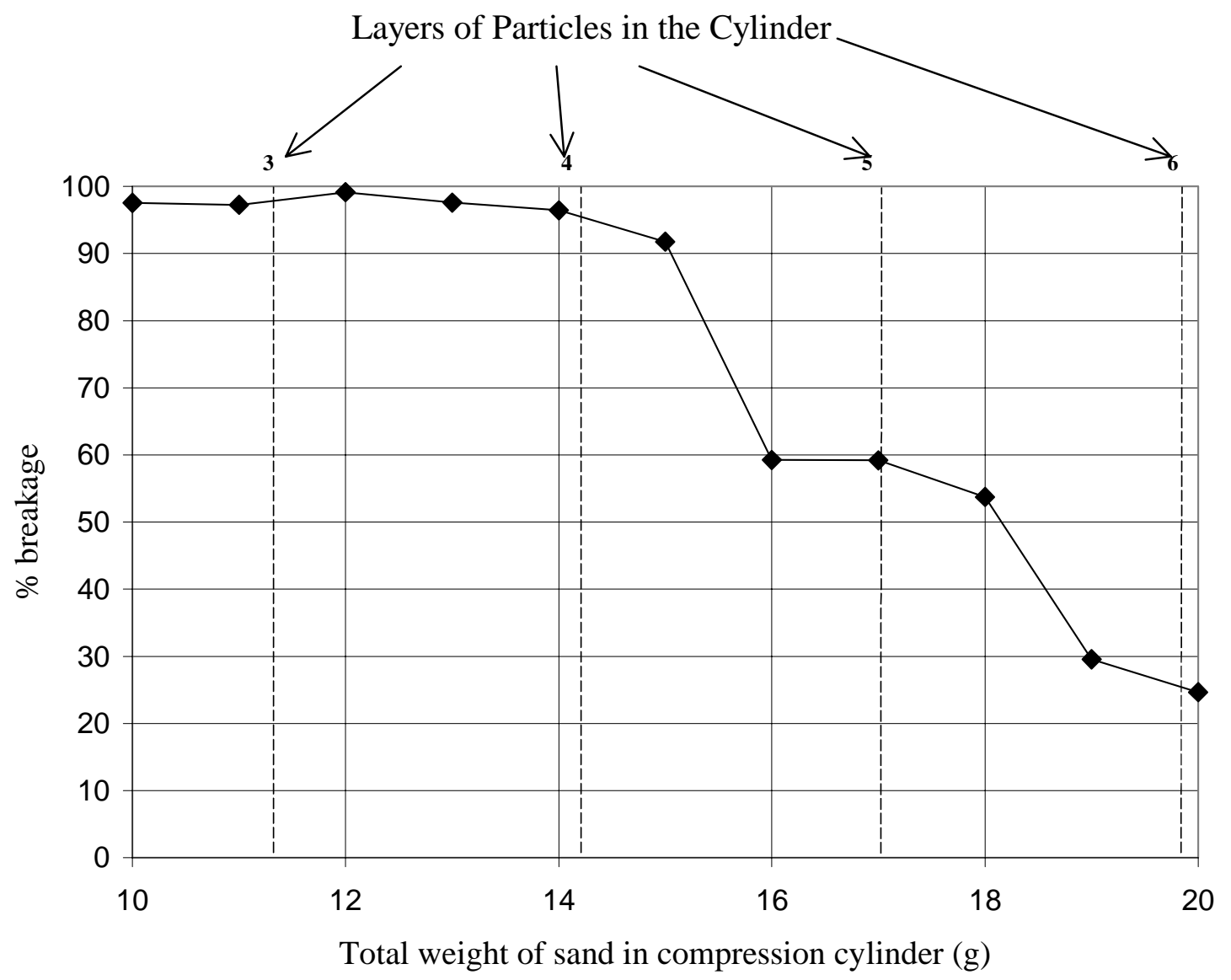

Figure 3.4: The percentage breakage of Nu-Pareil particles at different loadings in a bed of sand (2000 psi pressure, ratio of sand to Nu-Pareil is 100:1) 
titrant, a standardization procedure was used.

\section{Standardization of Thiosulfate Solution}

The primary standard, potassium iodate $\left(\mathrm{KIO}_{3}\right)$, was used to standardize the sodium thiosulfate solution. The standardization was conducted by dissolving $200 \mathrm{mg}$ $\mathrm{KIO}_{3}$ in $200 \mathrm{ml}$ of deionized water. A $5 \mathrm{ml}$ aliquot was removed to which $6.25 \mathrm{ml}$ of $20 \%$ potassium iodide $(\mathrm{KI})$ and $1.25 \mathrm{ml}$ of $6 \mathrm{M} \mathrm{HCl}$ was added. The resulting brown solution was immediately titrated to a faint yellow color, 5 drops of a starch indicator were added, and the solution was further titrated until it turned from a faint purple color to a clear end point. From the weight of the $\mathrm{KIO}_{3}$ added and the volume of the titrant added, the molarity of the thiosulfate solution was calculated. This was repeated three times to determine an average molarity.

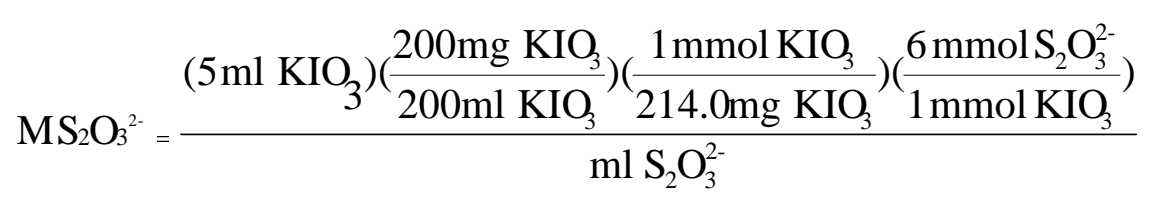

\section{Preparation of the Starch Indicator Solution}

1-2 grams of soluble potato starch were stirred into cold tap water to make a thick paste. This paste was slowly poured into $100 \mathrm{ml}$ of boiling water in which $1 \mathrm{~g}$ boric acid crystals were dissolved. Boiling was continued for 1 minute and then cooled to room temperature. The solution was transferred to a clean, stoppered bottle. The solution was discarded if it became milky or when it began to give a reddish color with iodine.

\section{Determination of \%AP breaker released}

The sample to be tested was added to $50 \mathrm{ml}$ of deionized water containing a small amount of surfactant. After a given soak time of 2, 5 and 10 minutes, a $5 \mathrm{ml}$ aliquot was 
removed, and transferred to a $50 \mathrm{ml}$ Erlenmeyer flask. $1.3 \mathrm{ml}$ of $20 \% \mathrm{KI}$ solution was added. The mixture was gently swirled, and the flask was sealed with a rubber stopper. The solution was allowed to stand for approximately $50 \mathrm{~min}$. Then $1.3 \mathrm{ml}$ of $6 \mathrm{M} \mathrm{HCl}$ solution was added, and the solution was allowed to stand for a further $10 \mathrm{~min}$. The solution was then titrated with the standardized sodium thiosulfate solution. The solution changed from a reddish brown to a yellow color. When it was a faint yellow color, 3 drops of the starch indicator were added. A dark purple color was formed. The solution was slowly titrated until the solution became clear. The volume of the titrant used was measured and entered into the following equation to determine \%AP released:

$$
\begin{gathered}
\mathrm{X} \text { mg AP released }=(\mathrm{ml} \text { of titrant })\left(\text { molarity of thiosulfate } \frac{\mathrm{mmol}}{\mathrm{ml}}\right)\left(\frac{1 \mathrm{mmol} \mathrm{AP}}{2 \mathrm{mmol} \text { thiosulfate }}\right) \\
\times\left(\frac{228.2 \mathrm{mg} \mathrm{AP}}{1 \mathrm{mmol} \mathrm{AP}}\right)
\end{gathered}
$$

Therefore, knowing the weight of AP in the core of the encapsulated sample $\mathrm{Y}$, we get

$$
\% \text { AP released }=\frac{\mathrm{X} \mathrm{mg} \mathrm{AP} \mathrm{released}}{\text { Y mg of AP in sample }} \times 100
$$




\section{Chapter 4}

\section{RESULTS AND DISCUSSION}

\subsection{POLYURETHANE COATINGS}

For the polyurethane tests, two coating levels $(8 \% \& 14 \%)$ were investigated. The results from leach and compression tests, using a loading of $11 \mathrm{~g}$ of coated particles and $0.11 \mathrm{~g}$ of proppant sand, are given in Figure 4.1. It can be seen from the figure that the leach rate at both coating levels was high, which was unacceptable. On the positive side, the release rates after compression are extremely high.

The high leach rates may be due to the non-uniformity of coating on the particles and/or the coatings being porous due to the presence of small bubbles in the coat formed by carbon dioxide evolution during the curing process. It is believed that a coating procedure, which would provide a more uniform coating on the particles, would reduce the leach significantly. This aspect needs to be examined further. Also, looking into higher levels of polyurethane coatings would be a worthwhile investigation.

Further tests using this system were not performed because of the inefficiency of the coating method. It was found that as the number of layers of polyurethane increased, the particles started forming large lumps, and this led to non-uniform coating. Hence, the emphasis was shifted towards acrylate coatings. 


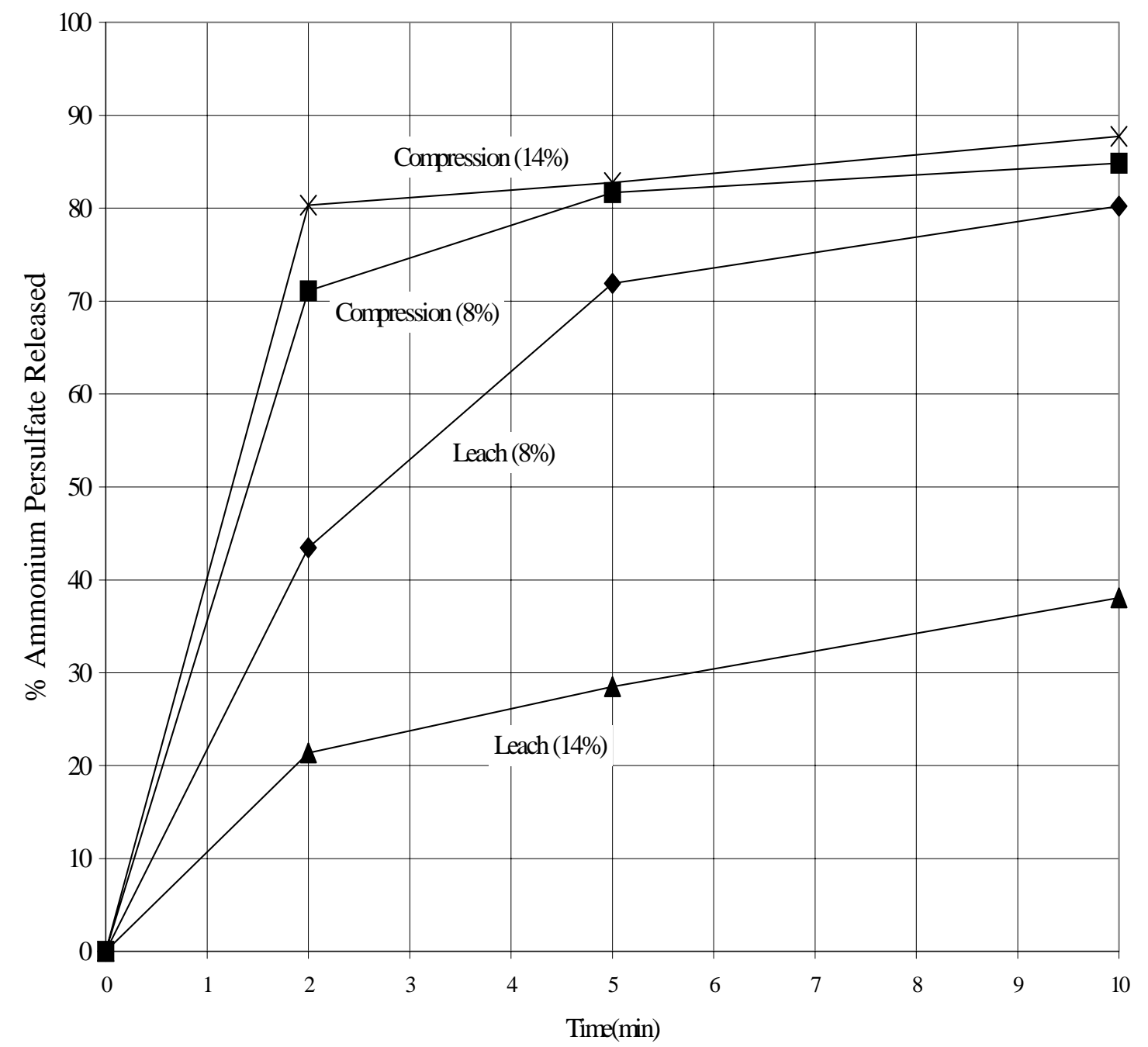

Figure 4.1: Compression and Leach Test Results for 2 Different Levels of Polyurethane Coating 


\subsection{ACRYLATE COATINGS}

Initial coating runs were carried out using acrylate coatings without any silica. The following sections discuss the effect of different variables on the compression and leach results of these coatings.

\subsubsection{Effect of Coating Level:}

The coating level on the particle was a significant factor in both the compression and leach tests. The $\%$ coating was a nominal value evaluated on the basis of the weight of the particles loaded in the bed and the amount of coating sprayed. This is not the actual coating amount, as there are some loss of coating material during the coating process.

A spray nozzle of diameter $0.61 \mathrm{~mm}$ was used for these coating runs. It was found that a significant amount of agglomerates was formed after the coating process. This issue will be discussed in detail in Section 4.2.2.

As seen in Figure 4.2, the \% AP released after 10 min., for leach and compression at different $\%$ coatings of acrylate varies significantly, with both decreasing with an increase in coating levels. This is because a higher coating level results in a more effective (thicker) moisture barrier for the particles. Also higher levels of coating are more difficult to break.

It was seen that the leach rates and release rates after compression reduced by about the same rate as the $\%$ of coating increased. 


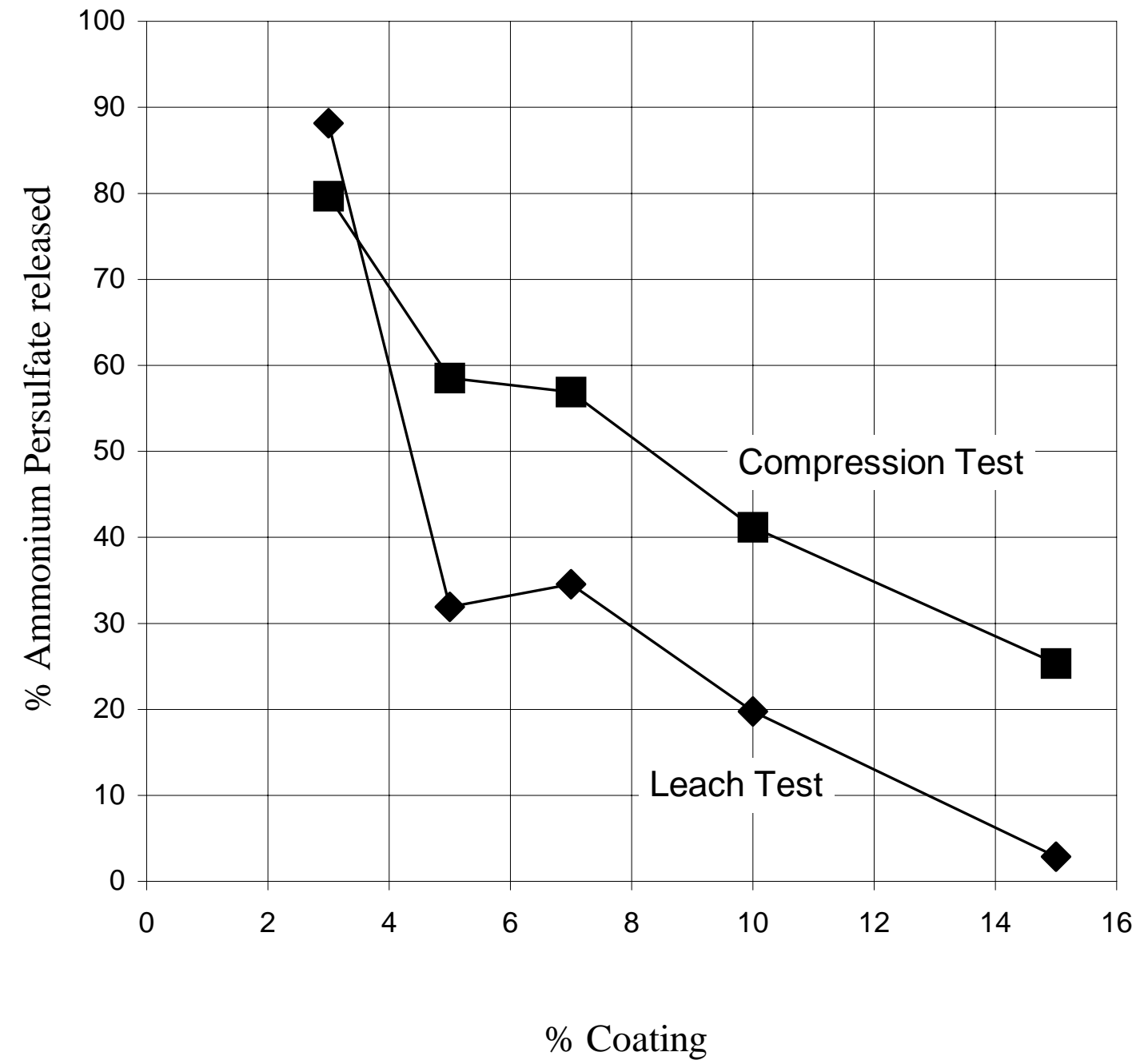

Figure 4.2: Amount of Ammonium Persulfate Released after 10 min. as a Function of $\%$ Coating of Acrylate 


\subsubsection{Effect of Nozzle Size on the Size Distribution of Coated Particles:}

Using two nozzles of different diameters, two different size distributions were obtained for particles coated with $15 \%$ acrylate. Nozzle diameters of $1.07 \mathrm{~mm}$ and $0.61 \mathrm{~mm}$ were used for Run 1 and Run 2 respectively. These results are compared with the size distribution of uncoated AP particles in Figure 4.3.

The difference in the size distributions between uncoated and coated AP was due to the formation of agglomerates of particles during the coating process. Since the acrylate coating took a long time to dry, the coated particles had a tendency to stick to each other while moving in the bed and thus formed agglomerates. In addition, larger nozzle diameters resulted in larger droplets and hence larger sizes of agglomerates.

The formation of agglomerates during coating is beneficial in the compression phase of this work. This is because it is easier to break agglomerates of coated particles than a single coated particle. This effect is shown in Section 4.2.3. The mechanism of formation and crushing of such agglomerates is discussed in Section 4.2.8.

The release rates for the two runs are shown in Figure 4.4. The tests were carried out on random samples of particles from each run. It was seen that the release rate after compression was greater for Run1, for which the particles/agglomerates were larger after coating. It is believed that larger-sized agglomerates are easier to break. Specifically, it is easier to shear and break the solid bridges of the agglomerate than it is to break a single coated particle. This effect is further addressed in Section 4.2.8 


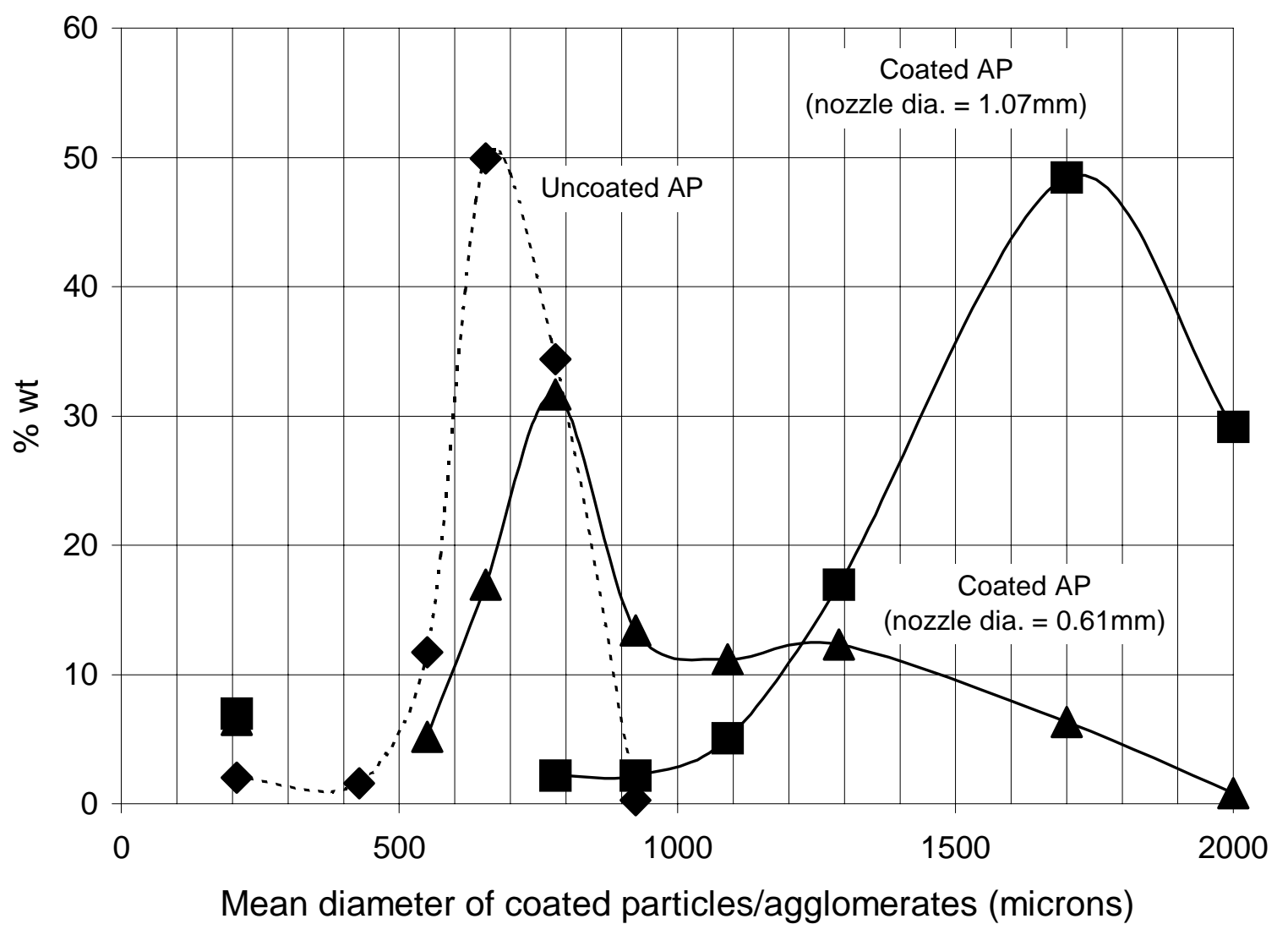

Figure 4.3: Size Distribution for Coating Runs with 15\% Acrylate using two Different Nozzle Diameters at the Same Atomizing Pressure (40 psi) 


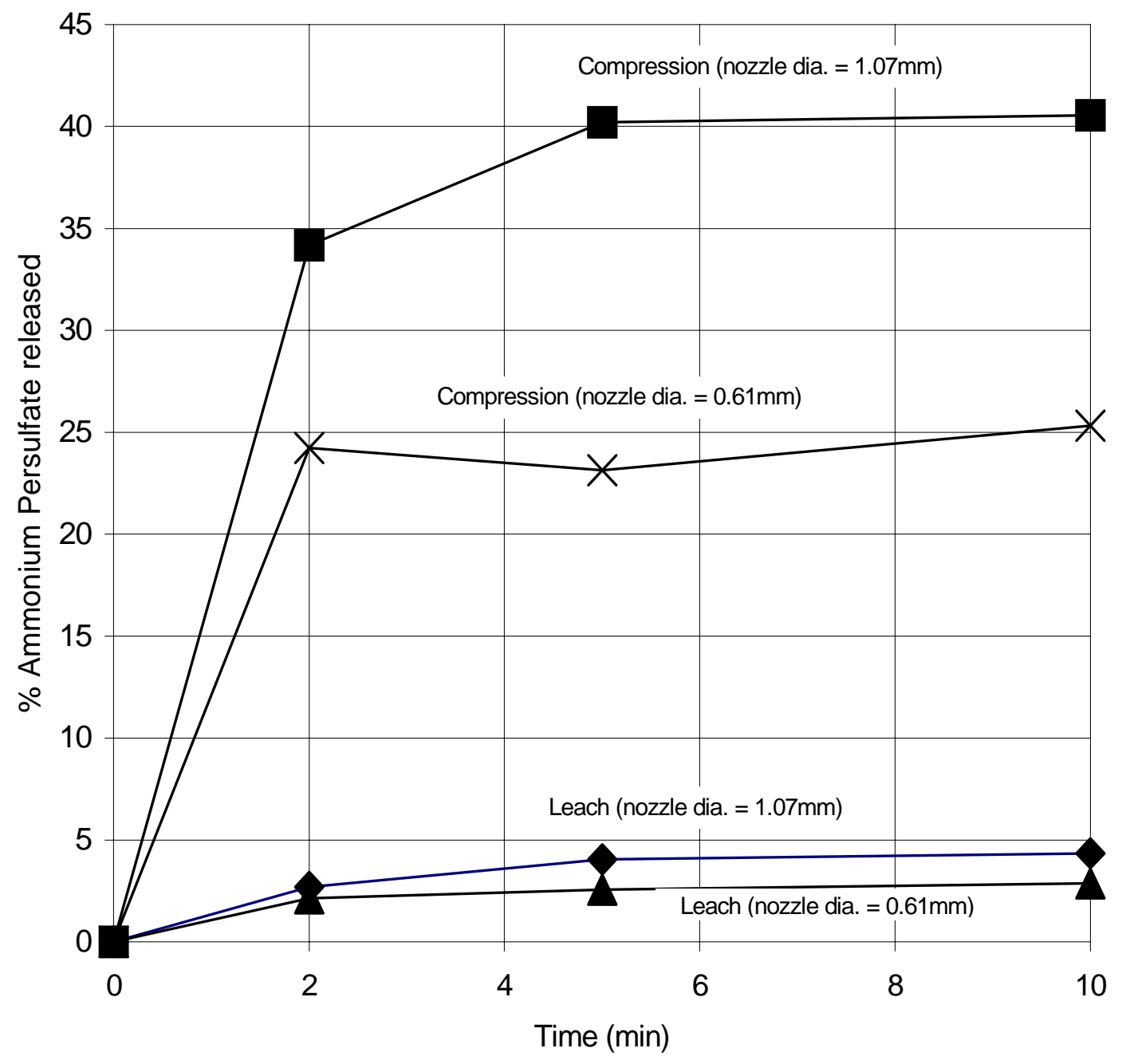

Figure 4.4: Release Rates for Different Size Distributions of 15\% Acrylate 


\subsubsection{Release Rates for Coated Particles/Agglomerates of Different Sizes}

For a $15 \%$ coating of acrylate (Run 2 above), the amount of AP released after 10 min. for different particle sizes was determined, and is shown in Figure 4.5.

It was seen that up to particle diameters of $1 \mathrm{~mm}$, the amount of AP decreased with an increase in particle size. It was seen that the coated particles of size smaller than $1 \mathrm{~mm}$ were single particles that were coated and did not consist of agglomerates. Sudsakorn [16] has shown that the amount of coating deposited in a fluidized bed coater increases with particle size. Hence, bigger particles have a higher level of coating as compared to smaller particles. As the coating level on a particle increases, the coating not only becomes more resistant to penetration by water, but it also becomes stronger. This accounts for the decrease in both the leach rates as well as the release rates after compression with an increase in particle size up to a particle diameter of $1 \mathrm{~mm}$. The size of the proppant sand was 18/40 mesh, which corresponds to particle diameters of $425 \mu \mathrm{m}$ to $1 \mathrm{~mm}$. Hence, coated particles with a diameter of up to $1 \mathrm{~mm}$ were of the same size as that of the proppant.

For particles larger than $1 \mathrm{~mm}$, the leach rate was very small and remained almost constant with particle size, whereas the release due to compression increased significantly with particle size. Coated particles of size greater than $1 \mathrm{~mm}$ were mostly agglomerate of smaller particles and typically contained between 6 to 10 particles. Hence it was the properties of the agglomerates that were being tested as opposed to that of single coated particles. It was found that larger the size of the agglomerates, the easier it was to break them. 


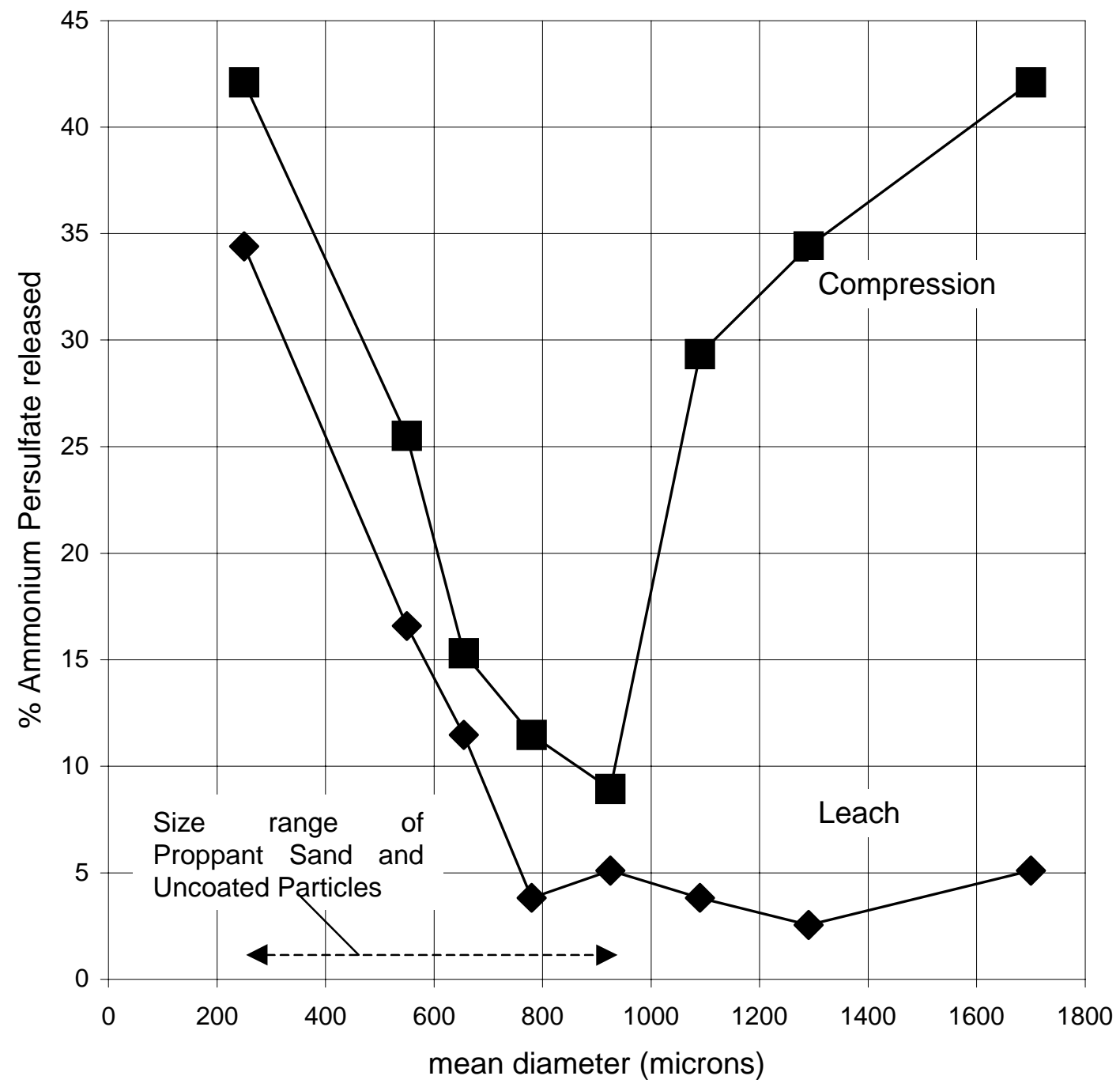

Figure 4.5: Release for Different Particle Sizes Produced in the Same Batch 
Thus we can see that for coated breaker particles that have the same size range as that of the sand, the effect of compression was not significant. However, coated particle agglomerates with sizes larger than that of the sand showed significant breakage under compression.

\subsubsection{Effect of Curing}

It was observed that curing the particles at elevated temperatures, immediately after coating, increased the resistance to water penetration. This is thought to be due to additional crosslinking within the coat. This reduced the leach rate but increased the release rate after compression.

The release rates for two samples of particles coated with $10 \%$ coating of acrylate, one cured at a temperature of $60^{\circ} \mathrm{C}$ and the other cured at room temperature for 2 days, is shown in Figure 4.6. The sample cured at $60^{\circ} \mathrm{C}$ showed a significantly higher release rate after compression, and a lower leach rate.

Though the cured samples gave more favorable results, the costs involved in terms of time and money to cure the samples on a large-scale did not justify the use of this process. Hence, this factor was not examined further for other coating compositions. 


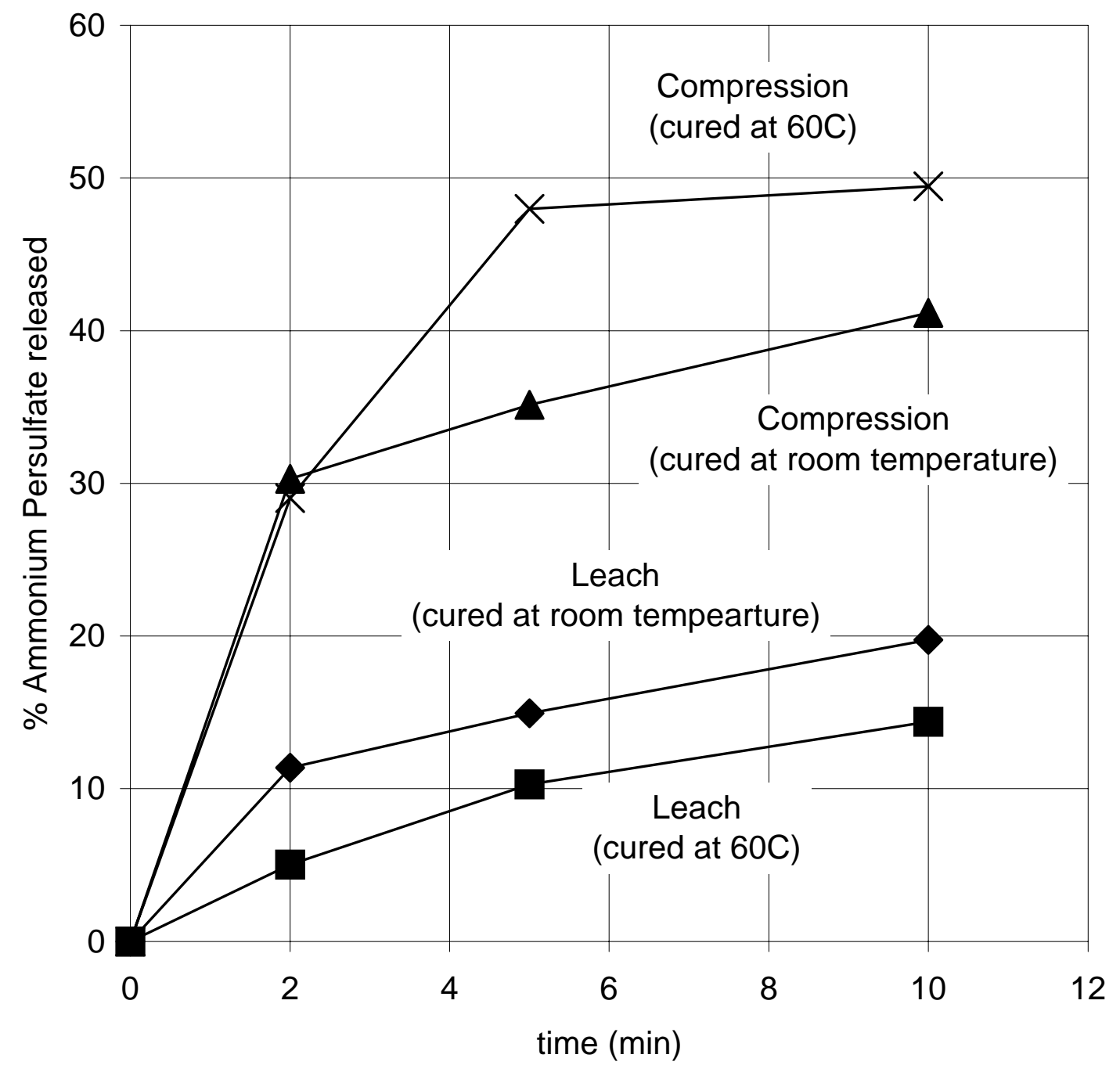

Figure 4.6: Effect of Curing Temperatures on Release for 10\% Coating of Acrylate 


\subsubsection{Effect of Silica in Coat}

It is postulated that the level of silica in the coat can affect the brittleness of the coat. Therefore, two different coating levels of acrylate (15\% and $25 \%$ by weight) were investigated. The weight $\%$ of the silica in the coat was varied from 0 to $80 \%$. The weight $\%$ of the silica in the coat was calculated as:

$$
\% \text { Silica }=\frac{\text { wt. of silica }}{\text { wt. of silica }+ \text { wt. of acrylate }} \times 100
$$

The weight of the crosslinker added was $3 \%$ of the total weight of the solids in the coat.

The compression and leach tests were carried out for a $1.4 \mathrm{~mm}-2 \mathrm{~mm}$ size cut of coated particles/agglomerates. Figure 4.7 shows the leach and compression results after 10 min. for $15 \%$ and $25 \%$ acrylate levels at different $\%$ levels of silica in the coat. From this figure, it is seen that at the $15 \%$ acrylate level, the $\%$ AP released went on increasing for both leach and compression, as the wt \% of silica in the coat increased. The amount of breakage on compression was maximum at the $60 \%$ silica level. At this level, the release after compression was about $75 \%$. The release for the leach test was $15 \%$. For the applications of this research, leach rates greater than $5 \%$ would be unacceptable. At the $80 \%$ silica level, the release rates after compression decreased, while that after leach increased significantly to $65 \%$.

A similar trend was also observed for the $25 \%$ acrylate level. Again, the maximum compression was obtained at the $60 \%$ silica level. At this level, the release after compression was up to about $67 \%$. The release for the leach test was $3.5 \%$. This coating condition gave the largest difference between the crushed and uncrushed release. 


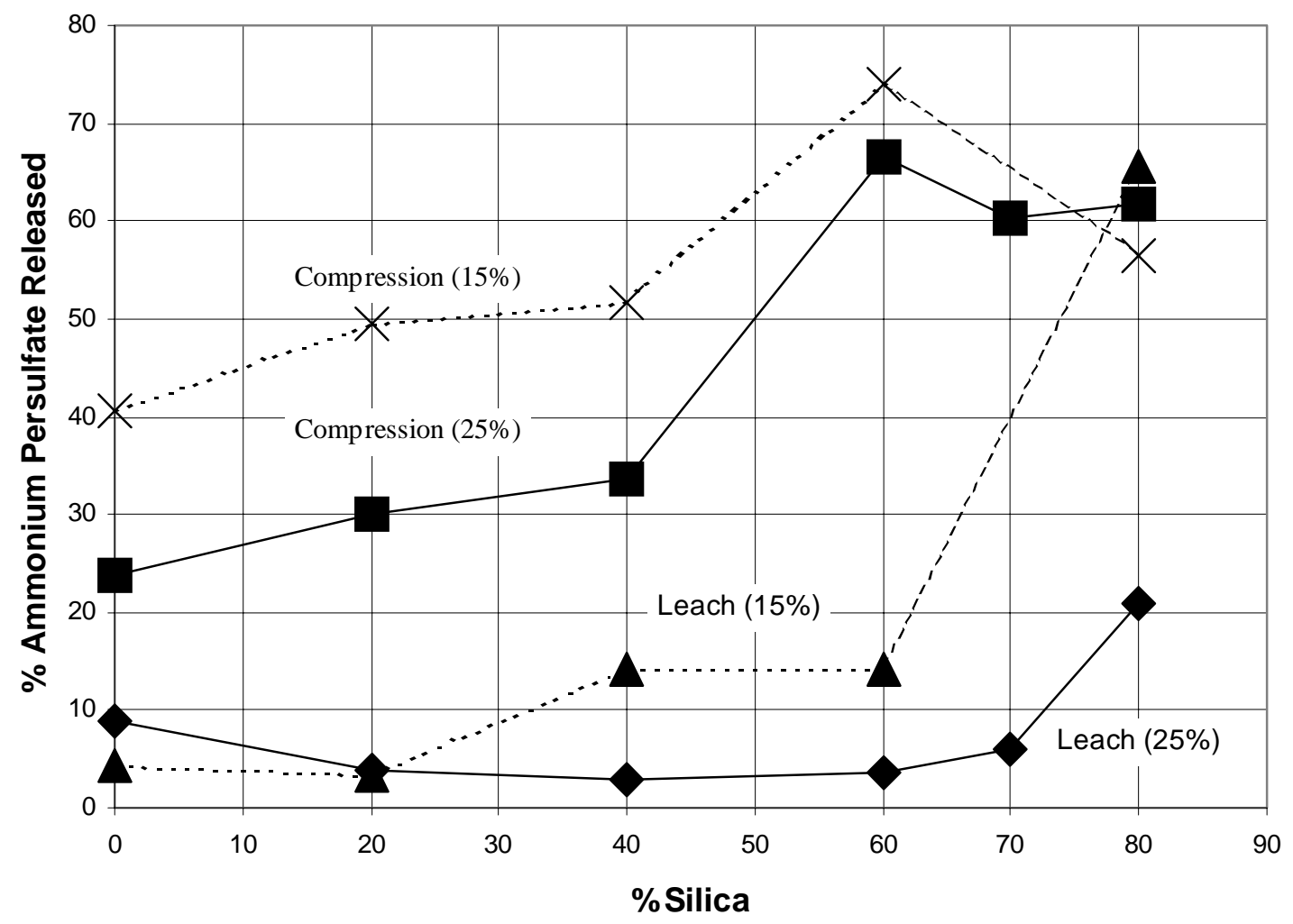

Figure 4.7:Amount of Ammonium Persulfate released after 10 min for leach and compression as a function of $\%$ silica in the coat at $15 \%$ and $25 \%$ acrylate levels 
Thus, there appears to be an optimum at around $60 \%$ by weight of silica for both coating levels of acrylate. The formulation that yielded the best results was:

Acrylate $=25 \%$ by weight of (Uncoated AP + Acrylate $)$

Silica $=60 \%$ by weight of (Acrylate + Silica $)$

Crosslinker $=3 \%$ by weight of $($ Acrylate + Silica + Crosslinker $)$

To understand these results, samples of size $5.5 \mathrm{~cm} \times 1 \mathrm{~cm} \times 0.2 \mathrm{~cm}$ were prepared from dispersions containing 25\% acrylate and different compositions of silica (ranging from 0 to $80 \%$ ). The initial aim of this procedure was to carry out strength tests on these samples using an Instron machine to determine the modulus and hence the brittleness at different silica compositions. However, it was found that the samples formed from compositions containing lower amount of silica (less than 60\%) were very flexible and would stretch to a high extent without being broken. Within the limitations of the Instron machine available to us, a suitable procedure to test the strength of these compositions could not be developed. However, on visually examining the samples, the following observations were made:

- Samples with $0 \%$ silica were extremely flexible, and could be stretched to a large extent without breaking. This ability to stretch went on decreasing as the amount of silica in the sample increased.

- Samples containing $60 \%$ silica did not stretch, and could not be broken easily by hand.

- Samples containing $70 \%$ silica were brittle, and could be easily broken by hand

- Samples containing $80 \%$ silica formed cracks and broke within the mould itself. The reason for this is that at such high levels of silica there is not enough acrylate to hold 
the silica particles together. This also explains the high leach, which is observed for the agglomerates coated with this composition.

\subsubsection{Release Rates for agglomerates coated with optimum formulation}

The persulfate released at different time intervals was measured for agglomerates coated with $25 \%$ acrylate and $60 \%$ silica. It is desired that the maximum amount of persulfate should be released in the minimum amount of time. During this time, the leach should be as low as possible. This is shown in Figure 4.8. It can be seen that the release rate, after compression, was a maximum during the first 2 minutes. After $2 \mathrm{~min}$, the release reaches a saturation level. The amount of AP released in leach was very low (less than $4 \%$ ) even after $10 \mathrm{~min}$.

The agglomerates coated with the same formulation were immersed in water for longer periods of time, and the persulfate released was measured. This is shown in Figure 4.9 . 


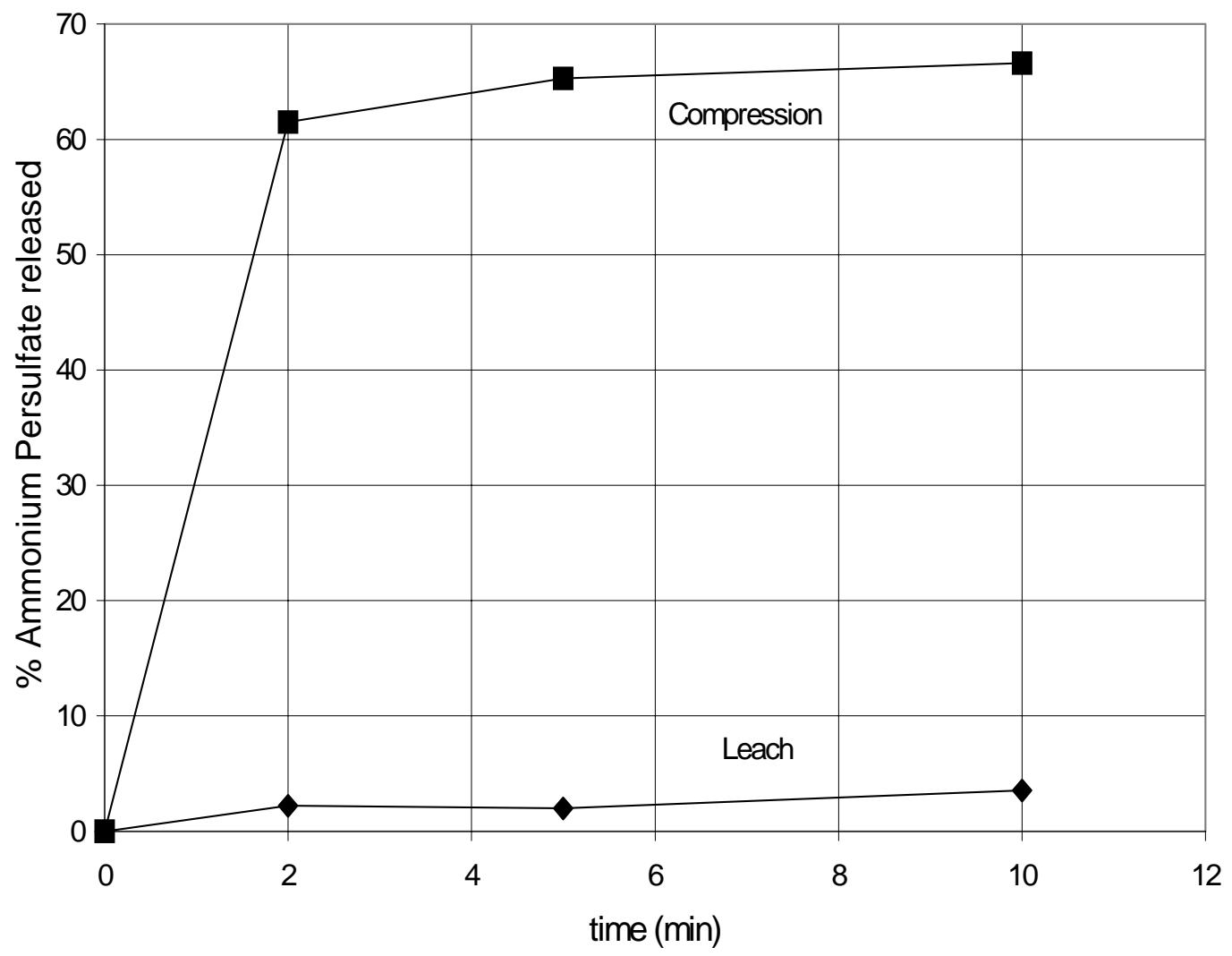

Figure 4.8: Amount of Ammonium Persulfate released with time for leach and compression tests of agglomerates coated with $25 \%$ acrylate and $60 \%$ silica. 


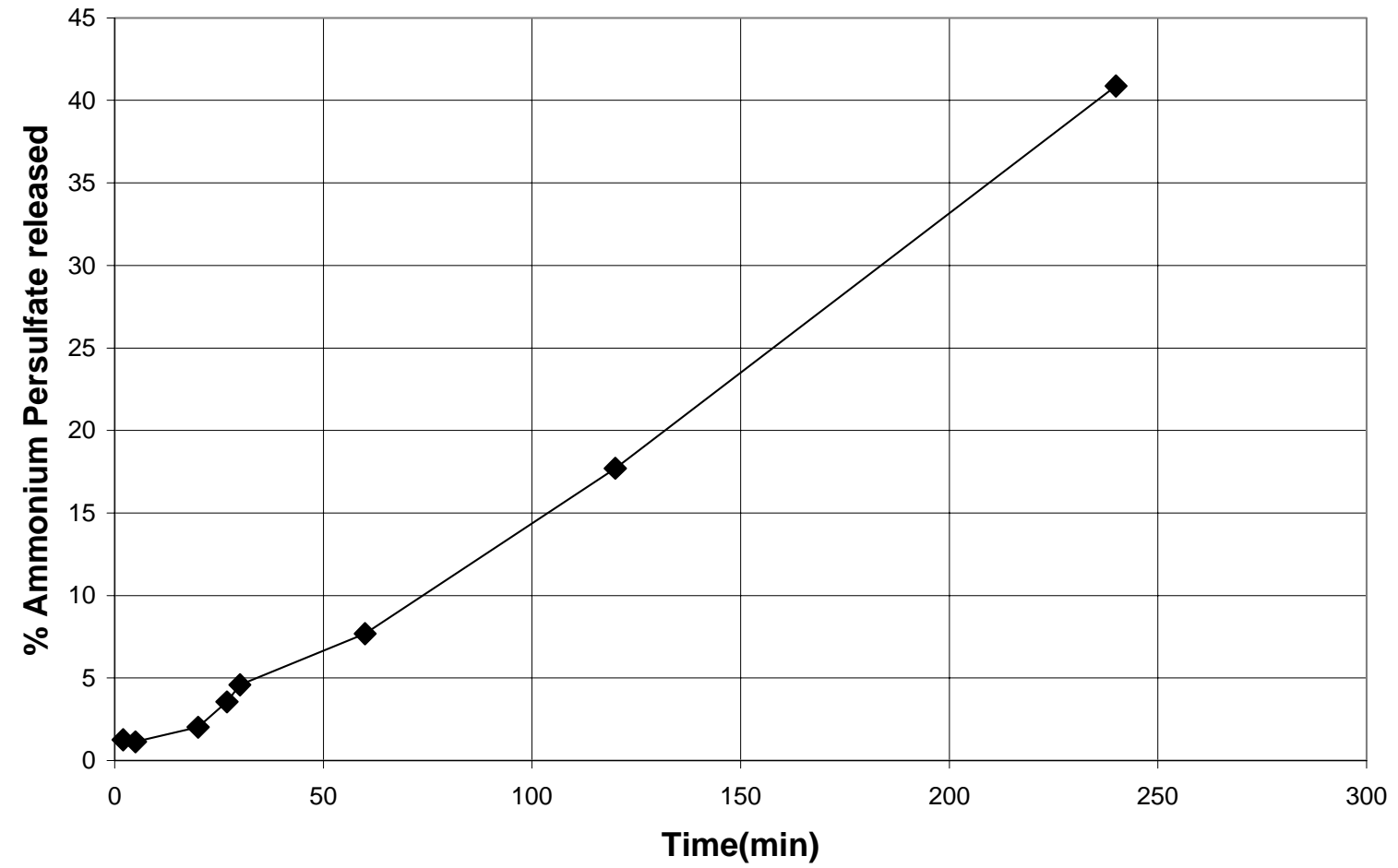

Figure 4.9: Amount of Ammonium Persulfate released for longer time periods under leach of agglomerates coated with $25 \%$ acrylate and $60 \%$ silica. 


\subsubsection{Comparison of Performance of Coated Agglomerates with Commercial Breakers}

In order to test the feasibility of using the coated agglomerates as breaker particles in the fracturing process, the performance of these agglomerates (coated with the optimum formulation) was compared with that of standard breakers which are presently used by Halliburton during the fracturing process. 2 standard breakers, Optiflo-II and Optiflo-III, which were obtained from Halliburton, were used for comparison. Both of these breakers are composed of ammonium persulfate particles coated with acrylate and silica. The formulation of the coat is proprietary, and information about it is unavailable. However, the coating is estimated to be approximately $33 \%$ by weight for Optiflo-II and $44 \%$ by weight for Optiflo-III. The persulfate released at different intervals of time as compared to the agglomerates is shown in Figure 4.10.

It can be seen that Optiflo-III gives extremely low leach (less than $0.5 \%$ after 1 hour). However, the release after compression is also very low (less than $2.5 \%$ after 1 hour). In case of Optiflo-II, the leach rate is almost the same as the agglomerates. However, the release after compression is much lower. The agglomerates give a much higher release rate after compression, while maintaining a leach of less than $7 \%$ in 1 hour and hence are considered to be superior in performance as breaker particles to Optiflo-II and Optiflo-III. 


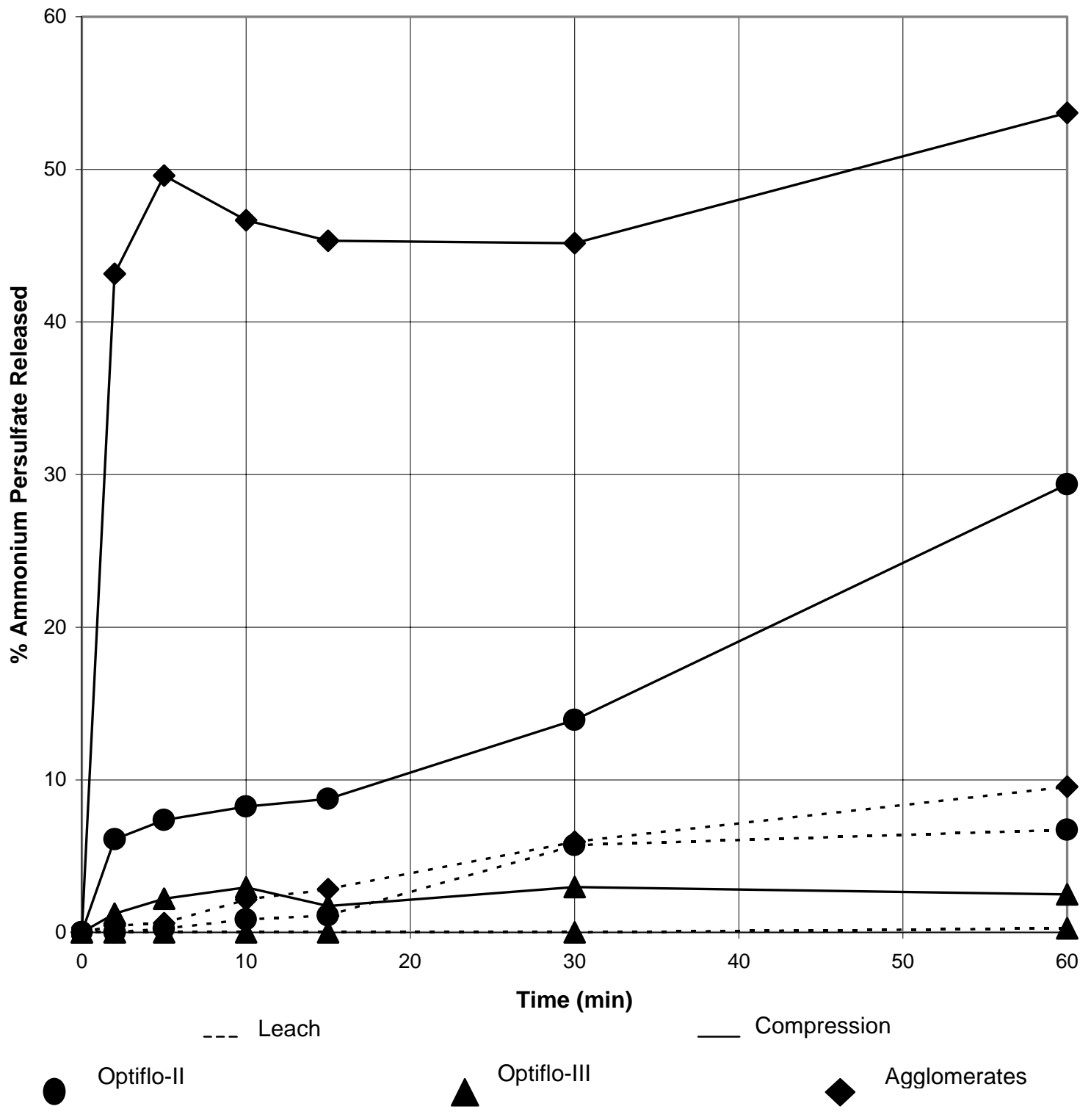

Figure 4.10: Performance of Agglomerates coated with Optimum Formulation compared to that of Standard Breakers 


\subsubsection{Mechanism of Formation and Crushing of Agglomerates}

For the operating conditions used in this research, the acrylate coating material took a relatively long time to dry. For this reason, once particles were coated with the acrylate they had the tendency to stick to each other and thus form agglomerates.

Figure 4.11 shows a digitized image (taken with a Sony Color Video Camera Model \# SSC-C350) of the agglomerate formed during a coating run with $25 \%$ acrylate. Figure 4.12 shows a SEM image of a sliced portion of the agglomerate. From these images, it can be clearly seen that the acrylate bridges between the coated particles to form agglomerates.

To understand the mechanism of crushing of agglomerates, samples of the agglomerates, after being subjected to the compression test, were viewed under a microscope, and are shown in Figure 4.13. From the figure, it can be seen that the acrylate bridge between the particles remains intact. However the particles themselves are sheared, exposing a surface which leaches out on contact with water.

On the basis of the above images, the mechanism of formation and crushing of agglomerates is understood to be as shown in Figure 4.14. 


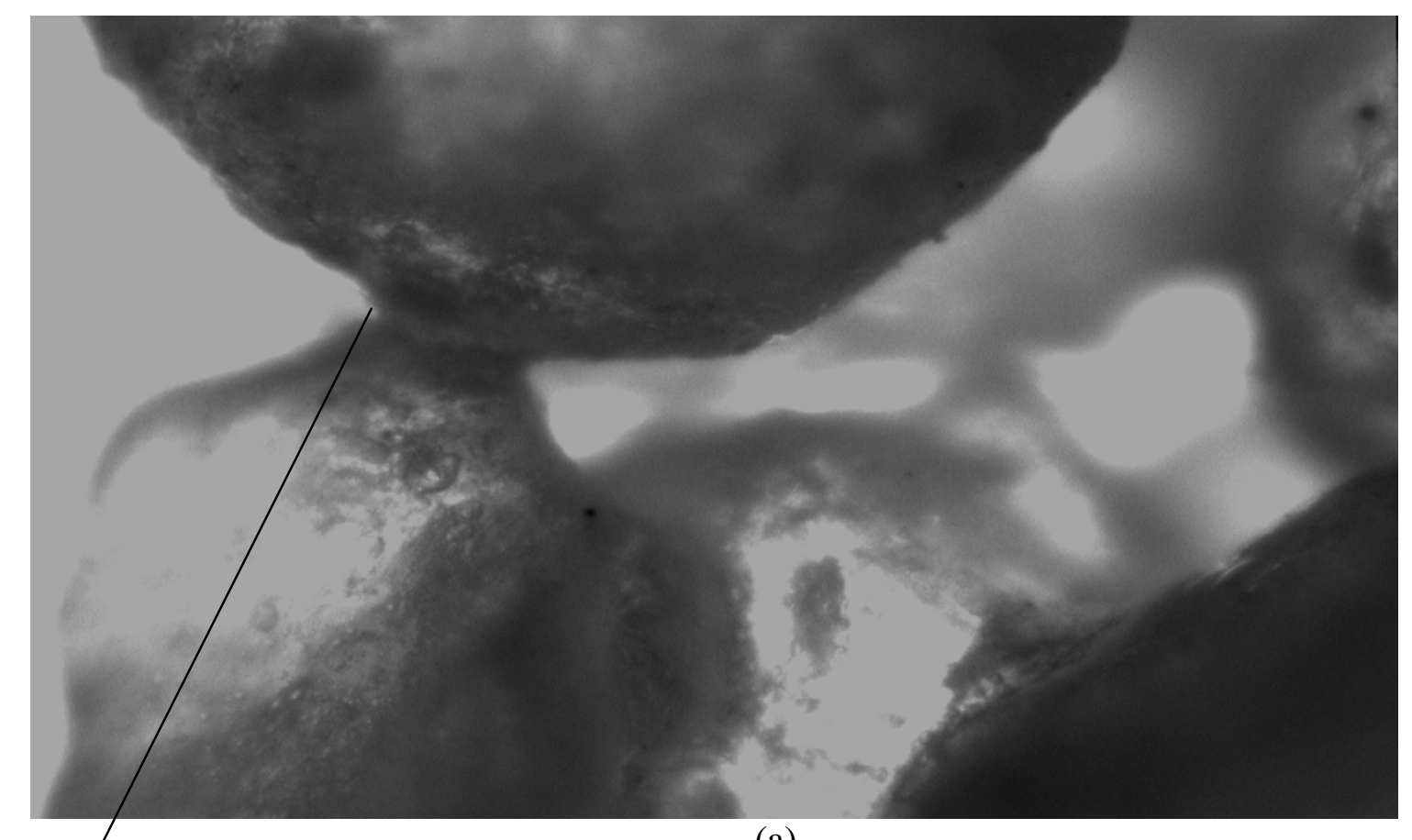

\section{Particle-particle bridge}

(a)

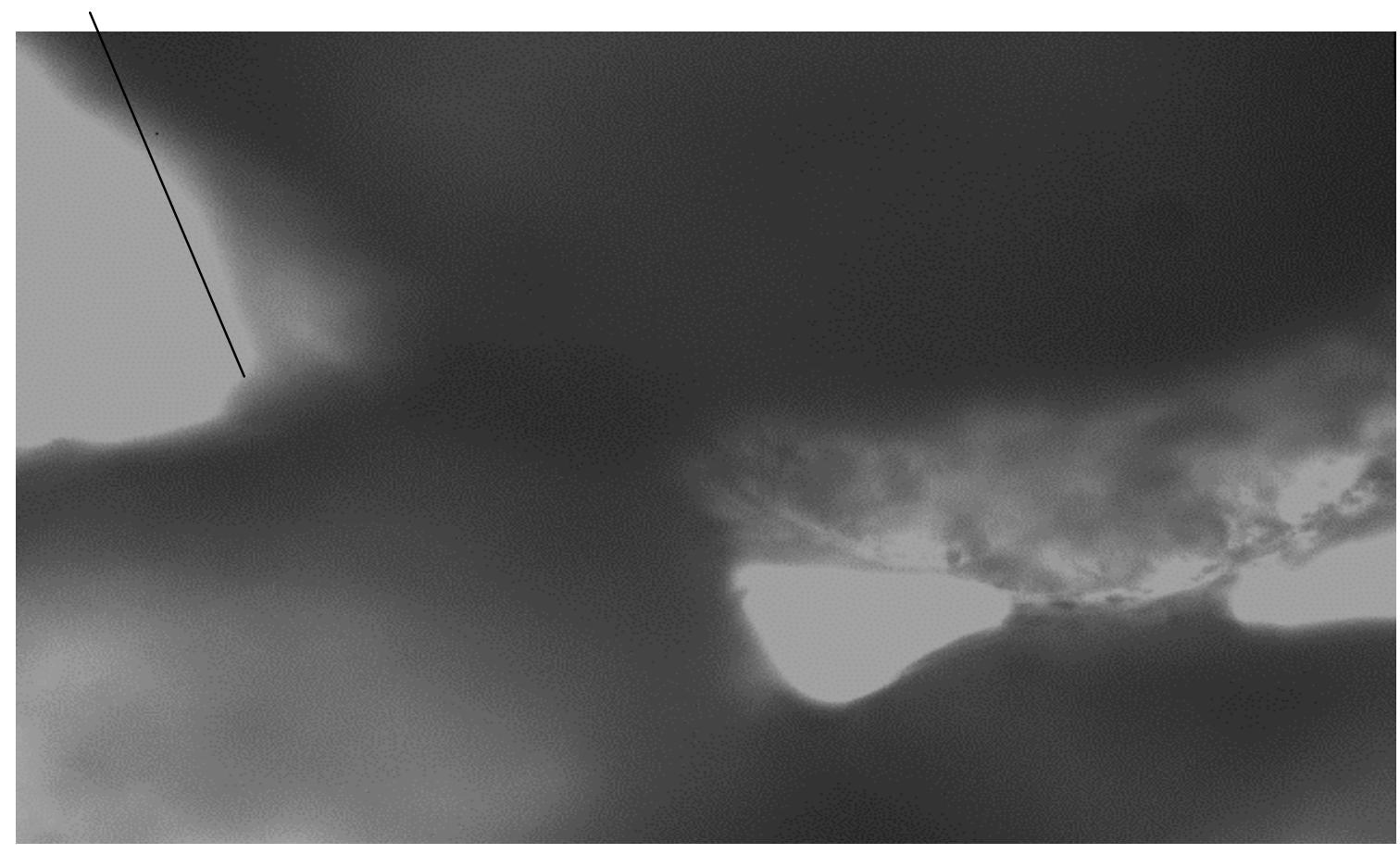

(b)

Figure 4.11: Agglomerate with 25\% acrylate coating 


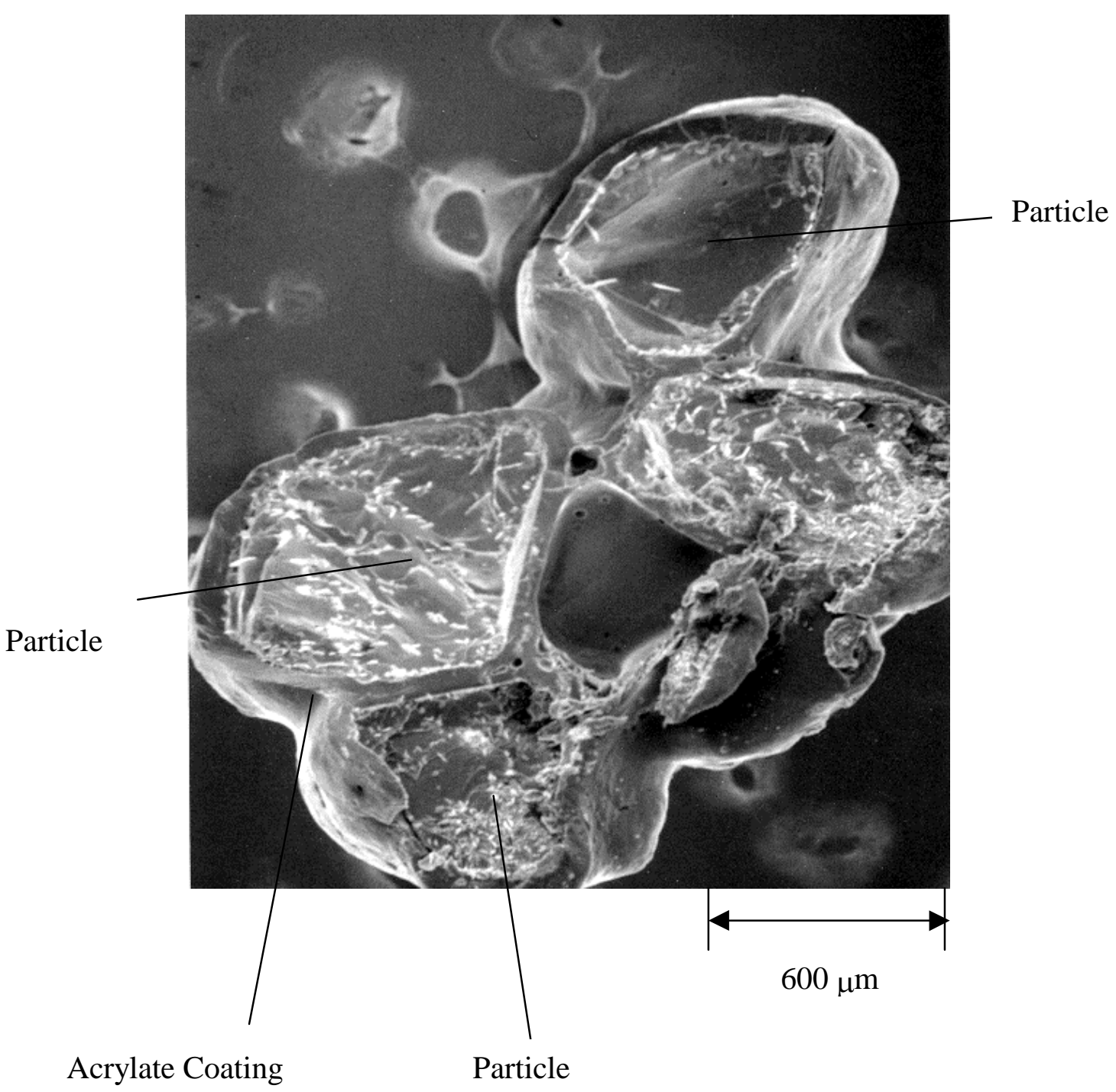

Figure 4.12: SEM Image of sliced agglomerate with 25\% acylate coating

$($ Magnification $=50)$ 


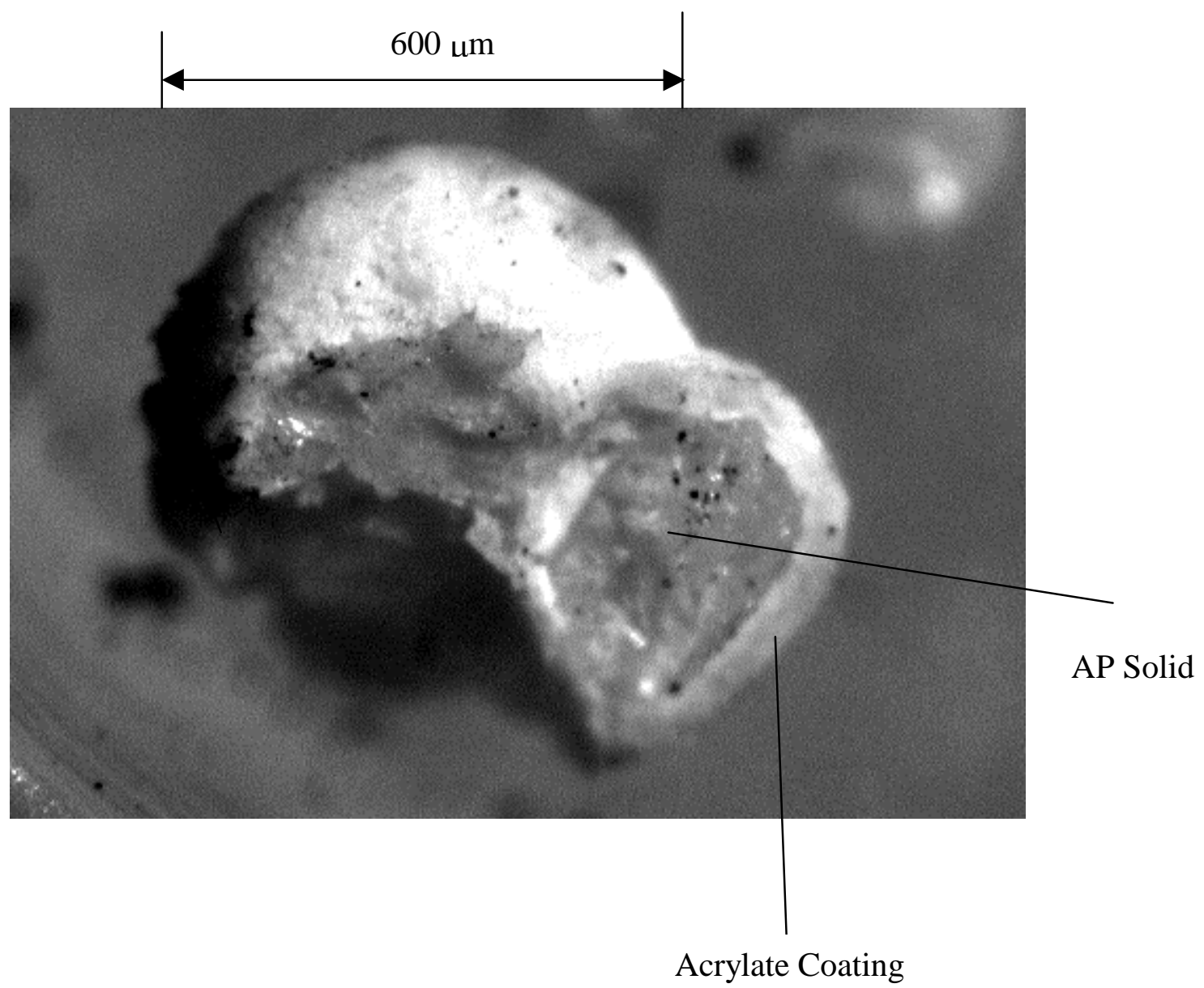

Figure 4.13: Crushed agglomerate with $25 \%$ acrylate coating, viewed under a microscope 


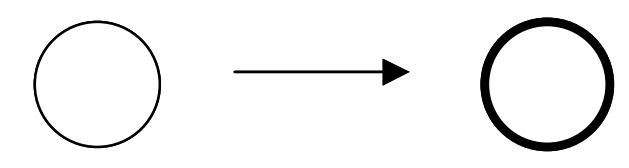

Uncoated AP

Coated AP

(a) Coating of Particle

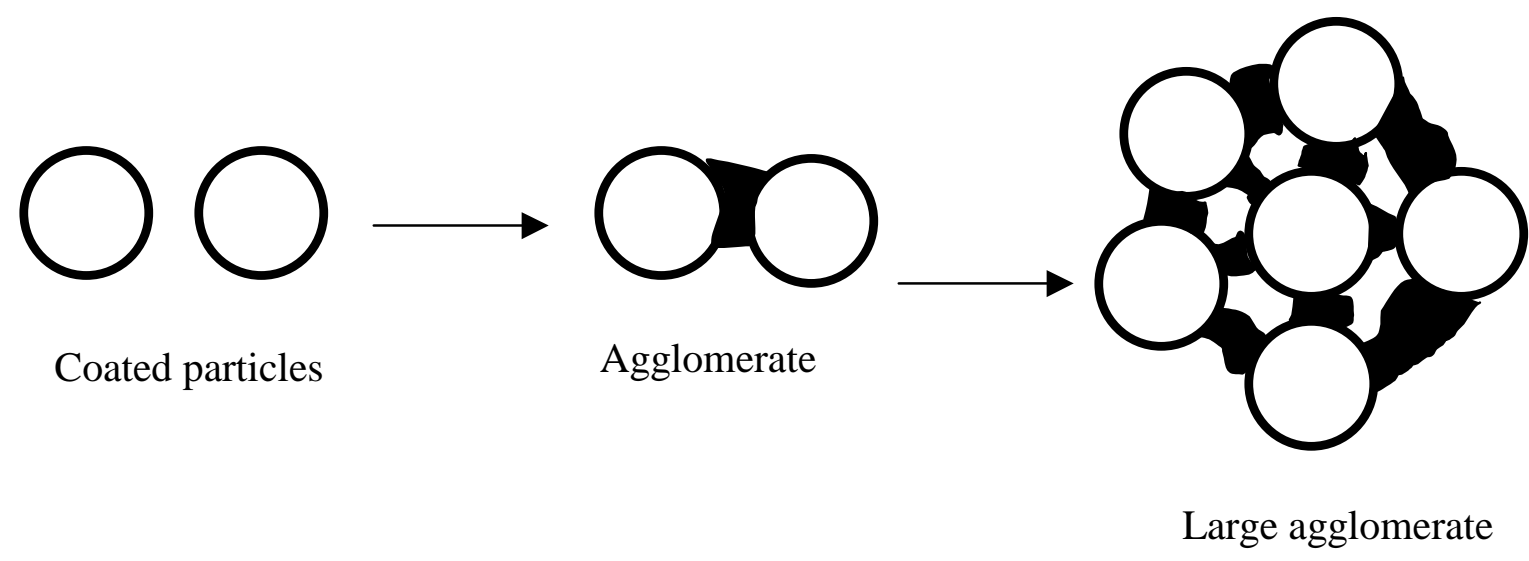

(b) Formation of agglomerates

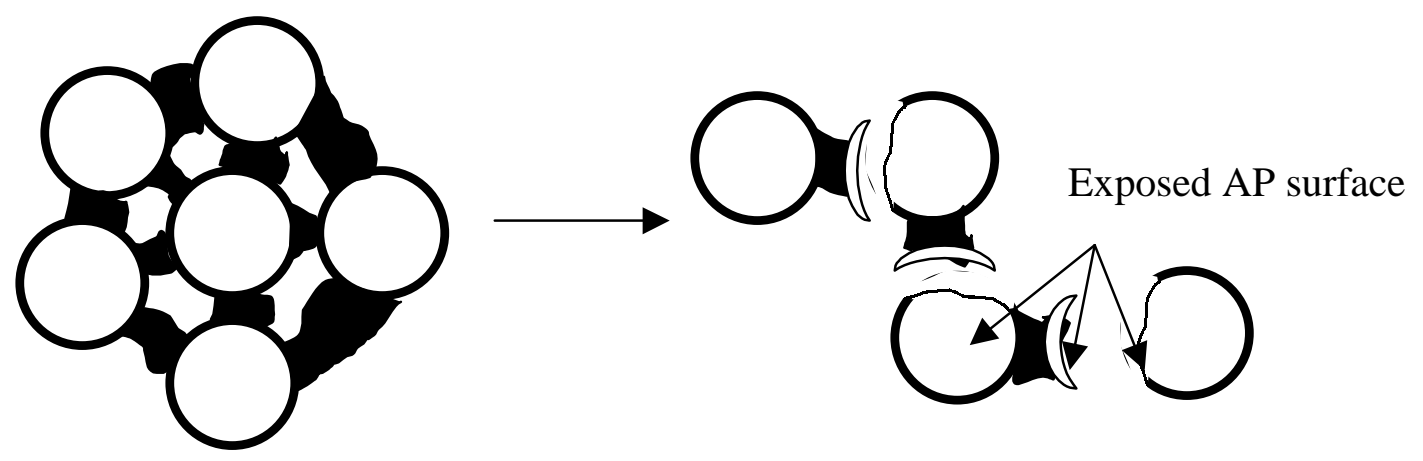

(c) Crushing of Agglomerates

Figure 4.14: Mechanism of Formation and Breakage of Agglomerates 


\subsection{9: Effect of Change in Concentration of Cross-Linking Agent}

It was believed that the level of cross-linking agent in the coat would affect the brittleness of the coat. Therefore, the effect of changing the amounts of the cross-linking agent (PFAZ-322) was examined. A coating of 25\% acrylate and 60\% Silica was used for these tests. The weight of the crosslinker in the coat was varied from 1 to $3 \%$ of the total weight of the solids in the coat.

Figure 4.15 shows the leach and compression results after $10 \mathrm{~min}$. for the three different levels of crosslinker.

It can be seen that there is no significant difference in the leach. However, there is significant difference in the release after compression. It was expected that the release rate would increase proportionally with an increase in crosslinker. This is because, as the crosslinking between the acrylate groups increases, it becomes increasingly difficult to break the acrylate bridge between the particles (as described in Section 4.2.8). However, it was observed, that though the release was the highest for $3 \%$ crosslinker, the release at $2 \%$ crosslinker level was lower than that at $1 \%$. This effect could not be explained and was not investigated further. All results in Sections 4.2.1 - 4.2.8 are for 3\% crosslinker and this is the recommended amount for this work. 


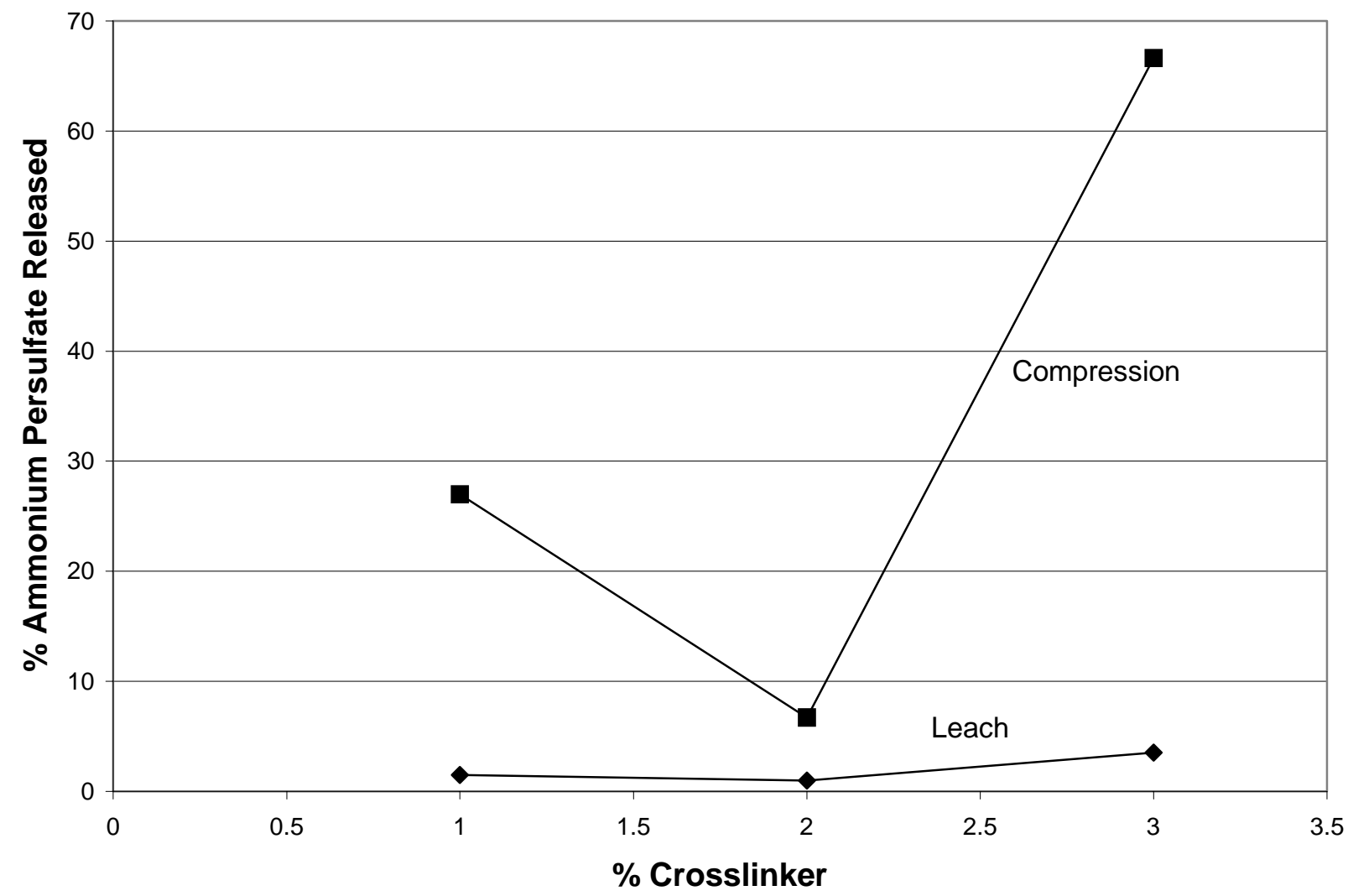

Figure 4.15: Amount of Ammonium Persulfate released after 10 min for leach and compression as a function of $\%$ crosslinker in the coat at $25 \%$ acrylate and $60 \%$ silica coating level 


\subsubsection{0: Effect of Draft Tube Height during the Coating}

It was found that the distance of the draft tube above the distributor plate had a significant effect on the release rate of the coated agglomerates.

The coating run of $25 \%$ acrylate, $60 \%$ silica and $3 \%$ crosslinker was carried out at 2 different draft tube heights of $1.2 \mathrm{~cm}$ and $3.5 \mathrm{~cm}$. The release for leach and compression for the 2 heights after $10 \mathrm{~min}$. is shown below:

$\underline{\text { Draft Tube height }}$

$3.5 \mathrm{~cm}$

$1.2 \mathrm{~cm}$ $\underline{\text { Leach }}$

$18.2 \%$

$6.24 \%$

\section{$\underline{\text { Compression }}$}

$65.2 \%$

$73.8 \%$

Hence, it was seen that the leach decreased significantly, while the compression increased slightly, at the lower draft tube height. This was more suitable for our application. Optimization of this variable plays an important role in obtaining particles with acceptably low leach rates. 


\section{Chapter 5}

\section{SUMMARY AND SIGNIFICANCE OF WORK}

\subsection{SUMMARY OF WORK}

The objective of this research was to find a chemical formulation that would provide a brittle and moisture-resistant coating for breaker particles. Breaker particles are viscosity reducing agents that are used in the fracturing process. The breaker that was investigated in this research was ammonium persulfate. The coatings that were investigated during the course of this work were polyurethane and acrylate coatings.

Coatings of polyurethane were achieved by mixing in a bottle, while acrylate coating was conducted in a bottom-spray fluidized bed.

The coatings were investigated for water resistance and susceptibility to brittle fracture. The coated particles were subjected to brittle fracture by compressing the particles along with proppant sand in a hydraulic compression machine. The amount of ammonium persulfate released, which was a measure of the water resistance of the coat (when uncrushed), or the fractional breakage (after compression), was measured using iodometric titration methods.

The polyurethane system that was investigated consisted of polymeric Diphenyl Methane Diisocyanate (MDI) and Diethylene Glycol (DEG). Particles coated with this formulation showed very favorable compression results. However, they had high leach rates, which need to be reduced significantly. This may be due to the non-uniformity of coating on the particles and/or the coatings being porous due to the presence of small bubbles in the coat formed by carbon dioxide evolution during the curing process.

The acrylate coatings were formulated from proprietary dispersions obtained from 
Halliburton, and an aziridine crosslinking agent. The addition of inert particles, like silica, was believed to increase the brittleness of the coat. Hence, during the final course of the work, silica particles were also added to the coat, and their effect on the brittleness of the coat was investigated.

The acrylate coating became sticky during the coating process, and resulted in the formation of agglomerates. These agglomerates must be within a particular size range to be used effectively as breaker particles. Agglomerates having a size range of $1.4-2 \mathrm{~mm}$ were investigated. Agglomerates larger than $2 \mathrm{~mm}$ were considered to be unacceptable as breaker particles because they would lead to non-uniform breaker concentration in the fracturing fluid.

Curing the agglomerates at elevated temperatures reduced the leach and increased the compression significantly. This was believed to be due to additional crosslinking at higher temperatures.

Tests were carried out for coated particles/agglomerates of different sizes produced in the same batch. The particles of size greater than $1 \mathrm{~mm}$, which were essentially agglomerates, were found to break upon compression more easily than single coated particles. It is postulated that it is easier to break an agglomerate of coated particles than it is to break a single coated particle.

Acrylate coatings of $15 \%$ and $25 \%$, with different wt $\%$ of silica, were examined for water resistance and fractional breakage after compression. The introduction of silica in the coat increased the breakage significantly. A coating formulation consisting of $25 \%$ acrylate coat, with $60 \%$ silica and $3 \%$ crosslinking agent gave the best results (largest difference in the $\%$ release between the crushed and uncrushed particles). For this 
formulation, a leach of less than $4 \%$ and compression release of over $65 \%$ was obtained after 10 minutes.

\subsection{SIGNIFICANCE OF WORK}

In this work, it has been shown that agglomerates of coated particles are easier to break than single coated particles. This is the most significant breakthrough that has been achieved in this research. It provides a novel solution to the seemingly opposing requirements of high water resistance and high susceptibility to brittle fracture for breaker particles. Agglomerates of coated breaker particles may hold promise of being used commercially as viscosity breakers for fracturing fluids. Finally a suitable coating formulation for these agglomerates has been investigated. 


\section{Chapter 6}

\section{RECOMMENDATIONS FOR FUTURE WORK}

\subsection{POLYURETHANE COATINGS}

Polyurethane coatings show promise, and warrant further investigation. The following aspects should be looked into, in order to increase the water resistance of the coating:

1. Increasing coating levels for the MDI-DEG system.

2. Using higher molecular weight polyurethane systems. One such system is:

Desmodur N 75 BA/X (Polymeric Hexamethylene Diisocyanate) \& Desmophen 651A-65 PMA (Saturated Polyester Polyol)

The above products are available commercially from Bayer Co.

3. Waterborne polyurethane coatings might be another option, as they can be coated using a fluidized bed, which would provide more uniform coating.

\subsection{ACRYLATE COATINGS}

The formation of agglomerates of acrylate coated breaker particles, which showed a high resistance to water as well a large fractional breakage during compression, was considered to be the main success of this research. The mechanism of the formation and breakage of these agglomerates needs to be investigated further, as this would provide valuable insight into methods that might increase the breakage after compression.

It is also believed that curing the coated particles under ultra-violet light promotes the crosslinking between the acrylate groups. This might lead to increased breakage after compression, as well as lower leach in water, and needs to be looked into. 
Finally further optimization of the coating formulation with respect to varying the amounts of acrylate, silica and crosslinker may be warranted if the \% of release upon compression must be increased.

\subsection{OTHER POLYMER SYSTEMS}

Other polymer systems might also provide moisture resistant brittle coatings. The two systems that are believed to be worth investigating are:

1) Polyvinylidene Dichloride: Polyvinylidene chloride copolymers (PVDC-CP) form extremely good barriers to water [17]. Water-based PVDC-CP latex like MorKote777, MorKote-5527, are manufactured by Morton and should be investigated. The main disadvantage of using PVDC is that it has a glass-transition temperature of 20$25{ }^{\circ} \mathrm{C}$. Hence at the high subterranean temperatures, it would soften, reducing the brittleness of the coat.

2) Polystyrene: Polystyrene (PS) is also believed to form a good moisture barrier. PS latex (Lucidene-370) is commercially available from Morton, and should be investigated. The main problem here is that PS has a high glass transition temperature of $100-105{ }^{\circ} \mathrm{C}$. This would be good for the actual applications, but it might cause problems during coating in a fluidized bed, as it is not a good film-former. A possible solution might be the addition of a plasticizer like Ethylene Glycol. 


\section{NOMENCLATURE \& ABBREVIATIONS}

$\begin{array}{ll}\text { AP } & \text { Ammonium Persulfate } \\ \text { MDI } & \text { Diphenyl Methane Diisocyanate } \\ \text { DEG } & \text { Diethylene Glycol } \\ \text { Scfm } & \text { standard cubic ft. per min. } \\ \text { Psi } & \text { lbf. per sq. in. } \\ \text { PVDC-CP } & \text { Polyvinylidene Chloride - Copolymers } \\ \text { PS } & \text { Polystyrene }\end{array}$




\section{REFERENCES}

1. Norman L. R. and S. B. Laramay; "Encapsulated Breakers and Method for Use in Treating Subterranean Applications"; US Patent No. 5,373,901, (1994).

2. Ely J. W.; "Fracturing Fluids and Additives"; Recent Advances in Hydraulic Fracturing; SPE Monograph Series, Volume 12, 131-146, Society of Petroleum Engineers, Richardson, TX (1989).

3. Burnham J. W., Briscoe J. E. and E. A. Elphingstone; "Methods and Additives for Delaying the Release of Chemicals in Aqueous Fluids"; US Patent No 4,202,795, (1980).

4. Walles W. E., Williamson T. D. and D. L. Tomkinson; "Method for Treating Subterranean Formations"; US Patent No 4,741,401, (1988).

5. King M. T.; "Method for Treating Subterranean Formations"; US Patent No 4,919,209, (1990).

6. Gupta S. D. V. and A. P. Cooney; "Encapsulation for Treating Subterranean Formations and Methods for the Use thereof'; US Patent No 5,164,099 (1992).

7. Nolte K. G; "Fracturing Fluid Breaker System which is Activated by Fracture Closure"; US Patent No 4,506,734, (1985).

8. Gulbis J., King M. T., Hawkins G. W., and H. D. Brannon; “Encapsulated Breaker for Aqueous Polymeric Fluids"; SPE Production Engineering; Society of Petroleum Engineers, February 1992.

9. Pondell R. E.; "From Solvent to Aqueous Coatings"; Drug Dev. Ind. Pharm.; 10(2), 191-202 (1984).

10. Squiller E. P and J. Rosthauser; "Catalysis in Aliphatic Isocyanate-Alcohol Reactions"; 
Modern Paint and Coatings; June 1987.

11. Norman L. and R. Turton; private communication, March 1998

12. Mehta A. M.; "Scale-Up Considerations in the Fluid-Bed processes for ControlledRelease Products”; Pharm. Technol.; Feb 1988.

13. Mehta A. M.; "Processing and Equipment Considerations for Aqueous Coatings"; Aqueous Polymeric Coatings for Pharmaceutical Dosage Forms; Marcel Dekker Inc.; New York (1989).

14. Oertel G.; Polyurethane Handbook; $2^{\text {nd }}$ Edition, Hanser/Gardner Publications, Inc., Cincinnati; (1993).

15. American Petroleum Institute RP 56: Procedures for Testing Frac Sand, Section 8, and "Recommended Frac Sand Crush Resistance Test”, Dallas, 1994.

16. Sudsakorn K.; "The Effect of Particle Size on the Amount of Coating Received During a Batch Fluidized Bed Coating Operation"; Master's Thesis, Dept. of Chemical Engineering, West Virginia University, 1999.

17. Gulbis J., King M. T. and T. D. A. Williamson; "Encapsulated Oxidizer for Hydraulic Fracturing Applications"; Proc., ACS Division of Polymer Chemistry; 30(1), (1989).

18. Norman L.; Procedure for Iodiometric Titration; January 1994. 


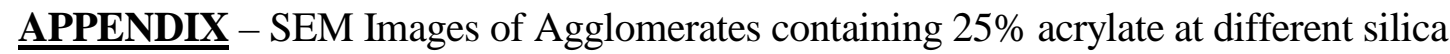
levels and various magnifications

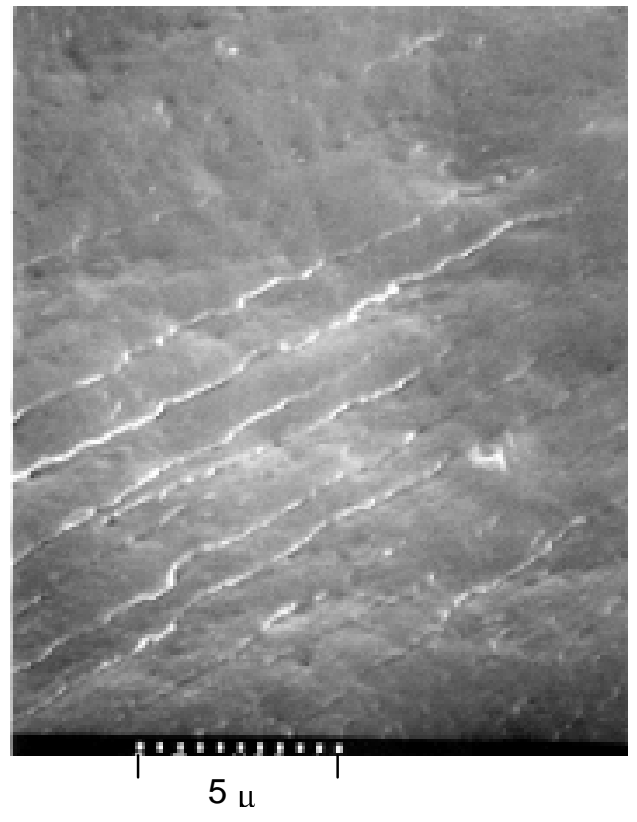

(a) $0 \%$ Silica

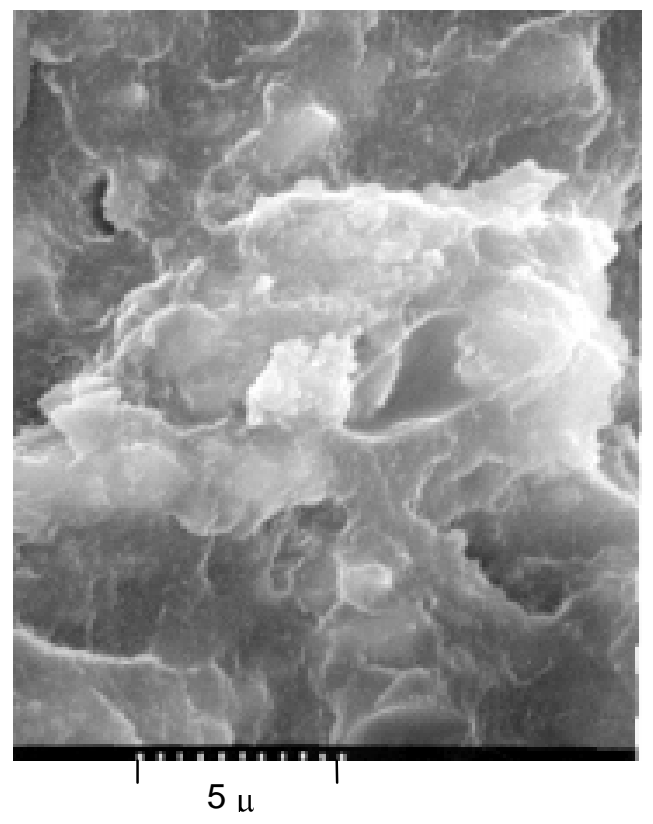

(b) $40 \%$ Silica

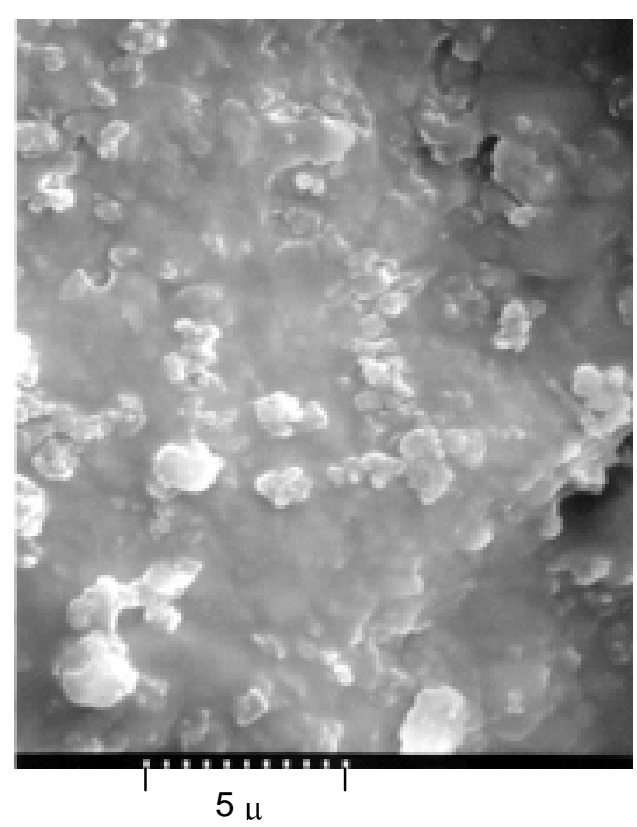

(c) $80 \%$ Silica

Figure A.1: Magnification $=6000 \mathrm{X}$ 


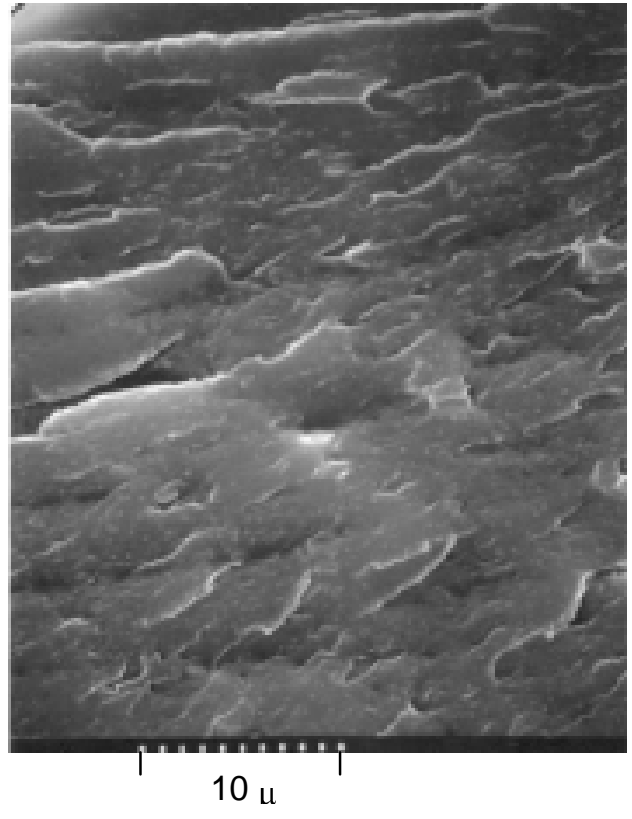

(a) $0 \%$ Silica

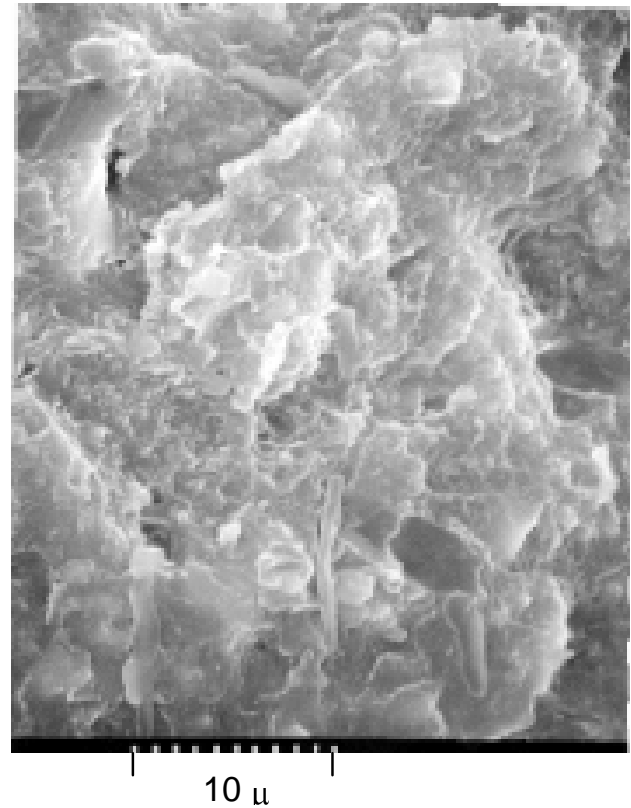

(b) $40 \%$ Silica

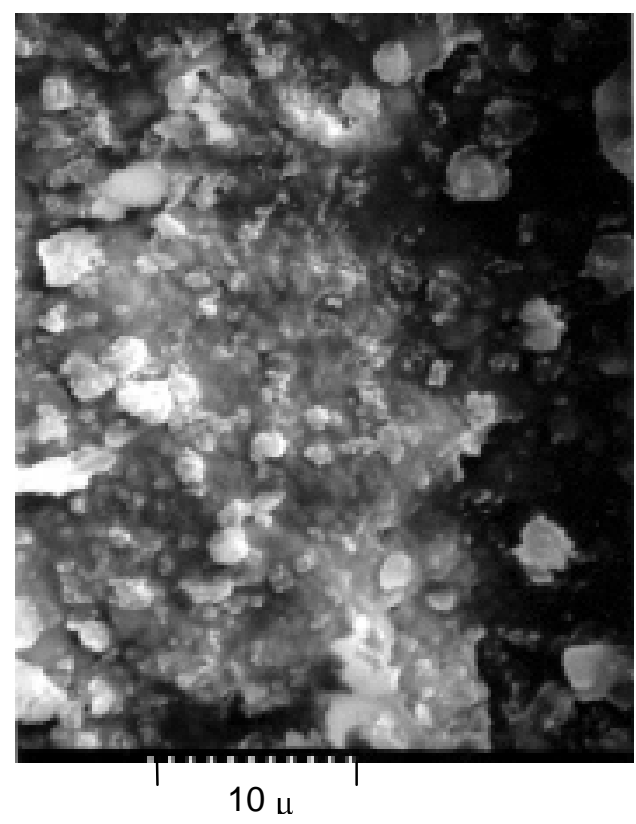

(a) $80 \%$ Silica

Figure A.2: Magnification $=3000 \mathrm{X}$ 


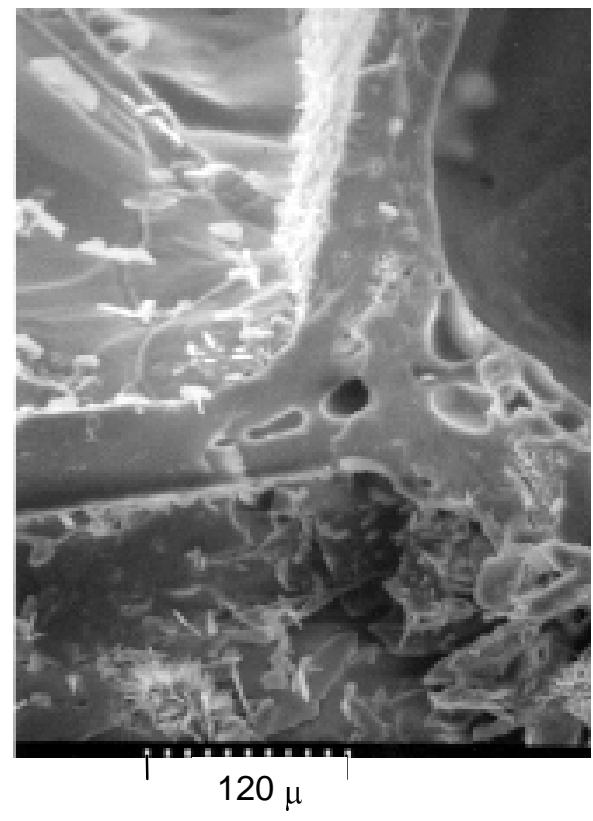

(a) $0 \%$ Silica

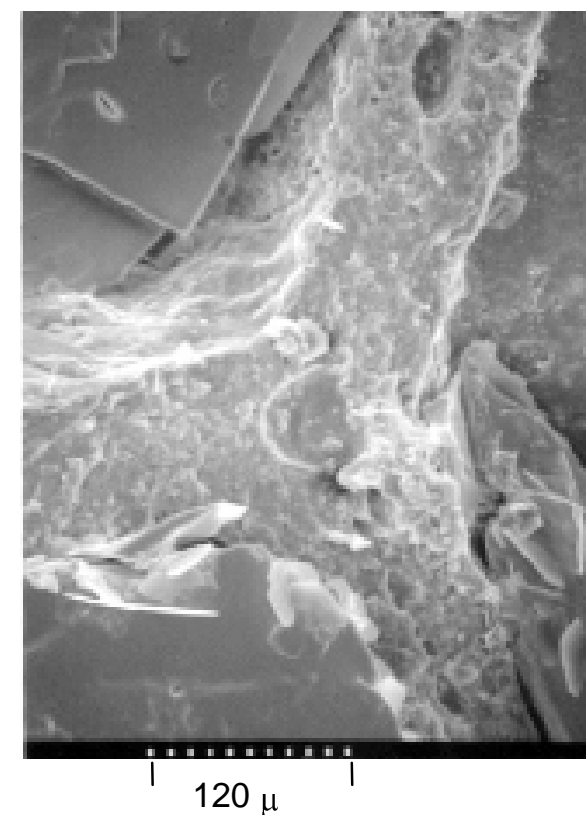

(b) $40 \%$ Silica

Figure A.3 Magnification $=250 \mathrm{X}$

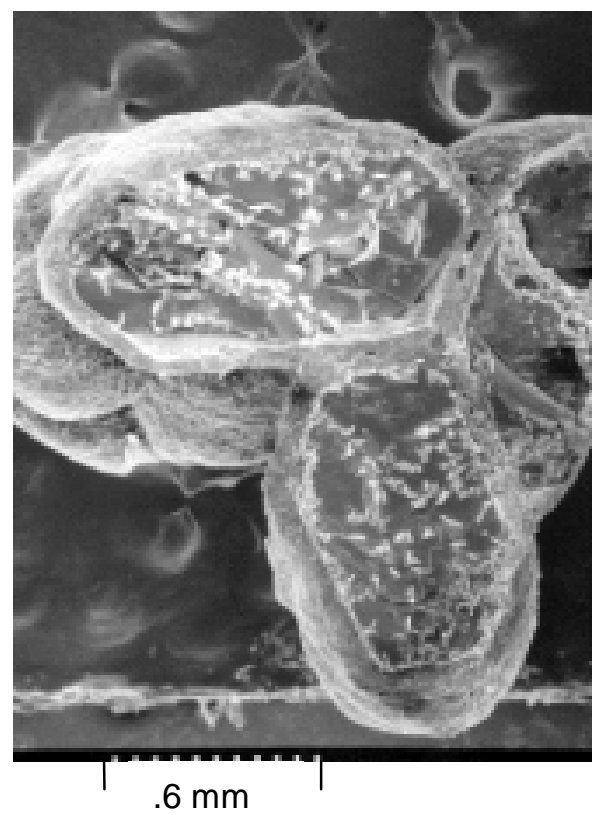

$40 \%$ Silica

Figure A.4: Magnification $=50 \mathrm{X}$ 